\title{
A Checklist of the Marine Plants from the Canary Islands (Central Eastern Atlantic Ocean)
}

\author{
R. J. Harouna*, M. C. Gil-Rodríguez ${ }^{\text {b }}$, J. Díaz de Castroa and W. F. Prud'homme van Reinec \\ a Department of Biology, Faculty of Marine Sciences, University of Las Palmas de Gran Canaria, 35017 Las Palmas, Spain \\ b Department of Plant Biology, Universidad of La Laguna, 38071 La Laguna, Tenerife, Spain \\ c Universiteit Leiden Branch, Nationaal Herbarium Nederland, P. O. 9514, 2300 RA Leiden, The Netherlands \\ * Corresponding author: ricardo.haroun@biologia.ulpgc.es
}

Since the last century, the richness of the Canary Islands marine flora has attracted many botanists. Børgesen's seminal papers have been the main reference source for anyone interested in this flora. The first checklist of the marine macroalgae from the Canary Islands was published by Gil-Rodríguez and Afonso-Carrillo (1980a) with 434 macroalgal species being listed together with some dubious records. In recent years, the research efforts of local phycologists and the output of some scientific expeditions have yielded some new species and a large number of new records to the Canarian seaweed flora. The aim of this contribution is to compile an up-to-date catalogue of the marine plants for the Canary Islands (including cyanobacteria, macroalgae, seagrasses and fungi). The review of all published records of these marine plants takes into account the present taxonomic status and nomenclatural changes of the taxa concerned and rejects old and dubious records. After a careful review of the pertinent references and revision of live and preserved material, only valid records published before July 2001 are included in this checklist. Besides, each species is quoted for the islands in an east-west fashion to illustrate the distributional pattern of the species concerned.The marine flora of the Canary Islands is composed of 711 species, which are distributed as follows: 59 Cyanophycota, 385 Rhodophycota, 125 Chromophycota, 117 Chlorophycota, 3 seagrasses and 22 fungi. Compared to nearby regions, the Canarian archipelago comprises a much larger number of species. This high diversity appears to be the result of the combined effects of its geographic location, paleoclimatic events and the prevailing oceanographic conditions.

\section{Introduction}

Since the time of Bory de Saint-Vincent (1803), the marine algae of the Canary Islands have attracted many other botanists. Among them, the work of Montagne (1840) is noteworthy and particularly the publications of Børgesen resulting from his field collections in Tenerife and Gran Canaria Islands (1925-30) can be considered as milestones in Canarian phycological history. Børgesen's papers have been the main reference source for anyone interested in this marine flora. However, the knowledge of Canarian seaweeds has increased, particularly during the last two decades. The first checklist of the marine macroalgae from the Canary Islands was compiled by Gil-Rodríguez and Afonso-Carrillo (1980a), who reported 434 species of macroalgae (including 24 Cyanophyta) and some dubious records. In recent years, the combined research efforts of local phycologists and the output of some scientific expeditions, such as CANCAP Expeditions in the 1980s and the Heincke Expedition (Haroun et al. 1993, Prud'homme van Reine 1998), have yielded a large number of new records and several new species to the marine flora (Gil-Rodríguez and Haroun 1992, Nizamuddin 1995, Tabares et al. 1997, Afonso-Carrillo et al. 1998, Prud'homme van Reine 1998). Most of this information has been used by Afonso-Carrillo and Sansón (1999) in their analytical key for the Canarian marine plants. Nevertheless, that publication does not compile the distributional pattern of the species quoted and, especially, several recent new records are not quoted (i. e., Sansón et al. 2002).

The aim of this contribution is to present an up-todate catalogue of the marine plants of the Canary Islands (sensu lato, including cyanobacteria, macroalgae, seagrasses and fungi). A review of all published records of these marine plants takes account of the present taxonomic status and nomenclatural changes of the taxa concerned, and rejects old and dubious records. However, this list does not pretend to be a definitive catalogue, as new records continue to appear, especially from poorly studied habitats such as the exposed northern coasts or subtidal environments.

Aside from its intrinsic floristic value, this new checklist has two meaningful applications. On the one hand, detailed biogeographical studies of a certain marine area (such as the rich Macaronesian Archipelagos) are only possible when reliable lists of species are available. A better understanding of the relationship of the marine flora from the Canary Islands with those of nearby coastal areas such as the Warm Temperate Eastern Atlantic coasts, the Mediterranean 
Sea, the Tropical Western African coasts or the Caribbean Sea will be enhanced. On the other hand, future conservation efforts, through such a powerful management tool as Coastal Zone Management Programs, should take account of the high biodiversity of the marine biota (both flora and fauna) which is found on specific Canarian islands as well as individual sections of coastlines.

\section{Material and Methods}

The systematic arrangement of the macroalgal species, including the ordinal and lower classification schemes, mainly follows Silva et al. (1996). Also, we have adopted the new division (phylum) names Cyanophycota, Rhodophycota, Chromophycota (which includes the Phaeophyceae) and Chlorophycota as recommended by these authors. In some cases, the nomenclatural status suggested by Wynne's (1998) recent revision of the tropical and subtropical marine flora of the western Atlantic is adopted. In addition, new taxonomic data for specific taxa, such as Chroococcales (Komárek and Anagnostidis 1995, 1999), Oscillatoriales (Anagnostidis and Komárek 1988), Stigonematales (Anagnostidis and Komárek 1990), Dictyota (Hörnig et al. 1992a,b, 1993), Nemacystus (Jong and Prud'homme van Reine 1997), Liagora (Abbott 1990a,b, Kvaternik and Afonso-Carrillo 1995), the Laurencia complex including Osmundea/Chondrophycus (Nam et al. 1994, Nam 1999, Nam et al. 2000), Neosiphonia (Kim and Lee 1999) and Eupogodon (Jong et al. 1997) are also incorporated. Many taxa have annotations added to present our view of its systematic position. In accordance with Guiry (1997), descriptive, rather than typified class names are used (e.g., Phaeophyceae instead of Fucophyceae). The marine phanerogams are arranged following Thorne (1992) and the marine fungi species (including the lichens) are listed using the systematic arrangement of Hawksworth et al. (1995). Genera and species appear alphabetically within their assigned family or genera respectively. Although Brummitt and Powell's (1992) compilation of names of authors is followed, nomenclatural authorities are given in full, mainly due to the quite diverse abbreviation systems in use by phycologists.

After a careful review of the pertinent references (up to July 2001) and revision of live/preserved material, only valid records are compiled in this checklist. Literature used to compile this checklist include, among others, Afonso-Carrillo (1980, 1983 and 1988), Afonso-Carrillo and Gil-Rodríguez (1982), Afonso-Carrillo et al. (1983), Audiffred (1984), Viera-Rodríguez et al. (1987), Morales-Ayala and Viera-Rodríguez (1988), Sansón et al. (1991), Ballesteros et al. (1992), Gil-Rodríguez and Haroun (1992 and 1993), Haroun et al. (1993), Sansón and Reyes (1994 and 1995), Betancor Villalba et al. (1995), González-Ruíz et al. (1995), Pedersen and Kristiansen
(1995), Martín et al. (1996), Guadalupe-González et al. (1996), Tabares et al. (1997), Prud'homme van Reine (1998), Afonso-Carrillo et al. (1998), RojasGonzález and Afonso-Carrillo (2000c) and Sansón et al. (2002). All records were critically reviewed and herbarium specimens examined where appropiate. Most of the specimens are deposited in BCM, TFC and $\mathrm{L}$; with some older collections in $\mathrm{C}, \mathrm{PC}, \mathrm{PAD}$ and W. Herbarium abbreviations are from the "Index Herbariorum" (Holmgrem et al. 1990). Some records need to be confirmed by further sampling; those species are included in a separate list (taxa inquirenda) until new information is available to reject or confirm their presence in the Canarian coasts. Misidentifications and dubious records are omitted.

\section{Results}

A list of marine plants reported for the Canary Islands is given in Table I, together with an indication, when possible, of the specific island. The symbol + denotes that the species is present somewhere on the Canary Islands, but its precise location is unknown. The records for La Graciosa, Montaña Clara and Alegranza (Islets located north of Lanzarote Island) are compiled together with those of Lanzarote Island. In some cases, annotations are given with the species name to clarify the taxonomic status of the specific taxon or to provide more information about the species concerned in the Canary Islands.

\section{Discussion}

Compared to neighbouring marine floras, the Canary Islands have a much larger number of marine species (South and Tittley 1986, Lawson and John 1987, Ribera et al. 1992, Gallardo et al. 1993, Nielsen et al. 1995, Guiry 1997), the only exception being the tropical and subtropical western Atlantic flora (Wynne 1998). The high number of marine species appears to be the result of the combined effects of the geographical position of the archipelago, its prevailing oceanographic conditions and paleoclimatic events (Prud'homme van Reine and van den Hoek 1990, Silva 1992, Prud'homme van Reine 1998). However, the present list cannot be considered as a definitive catalogue as new taxa will be added as subtidal environments are better sampled (Ballesteros et al. 1992, Haroun et al. 1993, Sansón et al. 2002) or detailed studies are carried out on certain groups (e.g.. Ceramiaceae by Sansón and Reyes 1994, 1995, and Rhodomelaceae by RojasGonzález et al. 1994, Rojas-González and AfonsoCarrillo 2000a,b).

The Canary Islands have a rich flora with co-ocurrence of floristic elements from the Mediterranean Sea, the Tropical Western Atlantic Ocean (mainly the Caribbean Sea) and the Warm Temperate North Atlantic coasts (both European and American coasts). 
Table I. List of marine plants and their distribution in the Canary Islands (+ = Canaries, $\mathrm{L}=$ Lanzarote I., F = Fuerteventura I., $\mathrm{C}=$ Gran Canaria I., T = Tenerife I., G=Gomera I., P = La Palma I. and H = El Hierro I.)

\begin{tabular}{|c|c|c|c|c|c|c|c|c|}
\hline & + & $\mathrm{L}$ & $\mathrm{F}$ & $\mathrm{C}$ & $\mathrm{T}$ & G & $\mathrm{P}$ & $\mathrm{H}$ \\
\hline \multicolumn{9}{|l|}{ CYANOPHYCOTA } \\
\hline \multicolumn{9}{|l|}{ CYANOPHYCEAE } \\
\hline \multicolumn{9}{|l|}{ Chroococcales (1) } \\
\hline \multicolumn{9}{|l|}{ Chroococcaceae Nägeli } \\
\hline \multicolumn{9}{|l|}{ Chroococcus Nägeli } \\
\hline C. turgidus (Kützing) Nägeli (2) & & & $\mathrm{F}$ & $\mathrm{C}$ & & & & \\
\hline \multicolumn{9}{|l|}{ Dermocarpellaceae Ginsburg-Ardré ex T. A. Christensen } \\
\hline \multicolumn{9}{|l|}{ Cyanocystis Borzì } \\
\hline C. minimus (Geitler) Komarek et Anagnostidis & & & & $\mathrm{C}$ & & & & \\
\hline C. olivaceus (Reinsch) Komarek et Anagnostidis & & & & $\mathrm{C}$ & $\mathrm{T}$ & & & \\
\hline \multicolumn{9}{|l|}{ Dermocarpella Lemmermann } \\
\hline D. prasina (Reinsch) Komarek et Anagnostidis & & $\mathrm{L}$ & & $\mathrm{C}$ & $\mathrm{T}$ & & & \\
\hline \multicolumn{9}{|l|}{ Entophysalidaceae Geitler } \\
\hline \multicolumn{9}{|l|}{ Entophysalis Kützing } \\
\hline E. conferta (Kützing) F. E. Drouet et W. A. Daily & & & $\mathrm{F}$ & $\mathrm{C}$ & $\mathrm{T}$ & & & \\
\hline E. deusta (Meneghini) F. E. Drouet et W. A. Daily & & $\mathrm{L}$ & $\mathrm{F}$ & $\mathrm{C}$ & $\mathrm{T}$ & $\mathrm{G}$ & & $\mathrm{H}$ \\
\hline \multicolumn{9}{|l|}{ Hydrococcaceae Kützing } \\
\hline \multicolumn{9}{|l|}{ Placoma Schousboe ex Bornet et Thuret } \\
\hline P. vesiculosa Schousboe ex Bornet et Flahault & & & & & $\mathrm{T}$ & & & \\
\hline \multicolumn{9}{|l|}{ Hyellaceae Borzì } \\
\hline \multicolumn{9}{|l|}{ Hyella Bornet et Flahault } \\
\hline H. caespitosa Bornet et Flahault & & $\mathrm{L}$ & & & & & & \\
\hline \multicolumn{9}{|l|}{ Pleurocapsa Thuret } \\
\hline P. fuliginosa Hauck & & & & & $\mathrm{T}$ & & & \\
\hline \multicolumn{9}{|l|}{ Merismopediaceae Elenkin } \\
\hline \multicolumn{9}{|l|}{ Aphanocapsa Nägeli } \\
\hline A. littoralis Hansgirg (3) & & & & $\mathrm{C}$ & & & & \\
\hline A. marina Hansgirg (4) & & $\mathrm{L}$ & & $\mathrm{C}$ & & & & \\
\hline A. salina Woronichin & & & & $\mathrm{C}$ & & & & \\
\hline \multicolumn{9}{|l|}{ Xenococcaceae Geitler } \\
\hline \multicolumn{9}{|l|}{ Xenococcus Thuret } \\
\hline$X$. acervatus Setchell et N. L. Gardner & & & & $\mathrm{C}$ & $\mathrm{T}$ & & & \\
\hline
\end{tabular}

\section{Oscillatoriales}

Schizotrichaceae Elenkin

Schizothrix Kützing ex Gomont

S. arenaria (Berkeley) Gomont

S. calcicola (C. Agardh) Gomont

S. mexicana Gomont

S. rubella Gomont

$\begin{array}{llll}\mathrm{L} & \mathrm{F} & \mathrm{C} & \mathrm{T} \\ \mathrm{L} & \mathrm{T} & \mathrm{C} & \mathrm{T}\end{array}$

$\begin{array}{llll}\mathrm{L} & \mathrm{F} & \mathrm{C} & \mathrm{T}\end{array}$

Phormidiaceae Anagnostidis et Komárek

Arthrospira Stizenberger ex Gomont

A. neapolitana (Kützing ex Gomont) F. E. Drouet

$\mathrm{L}$

Hydrocoleum Kützing ex Gomont

H. glutinosum (C. Agardh) Gomont

$\mathrm{C}$

Microcoleus Desmazières ex Gomont

M. chthonoplastes (Hornemann) Gomont

C T

M. codii Frémy

M. wuitneri Frémy

Phormidium Kützing ex Gomont

P. breve (Kützing ex Gomont) Anagnostidis et Komárek

$P$. corallinae (Kützing ex Gomont) Anagnostidis et Komárek

P. corium (C. Agardh) Gomont

P. nigroviride (Thwaites ex Gomont) Anagnostidis et Komárek

P. submembranaceum Gomont

P. subuliforme (Thwaites ex Gomont) Anagnostidis et Komárek

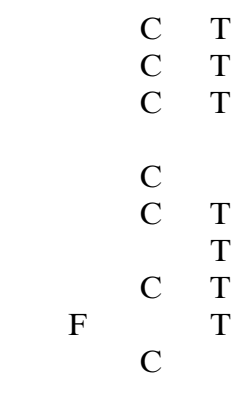

P. notarisii (Meneghini) Kützing ex Gomont

$\mathrm{L}$

C T

Spirulina Turpin ex Gomont 
Table I. Continued.

\begin{tabular}{|c|c|c|c|c|c|c|c|c|}
\hline & + & $\mathrm{L}$ & $\mathrm{F}$ & $\mathrm{C}$ & $\mathrm{T}$ & G & $\mathrm{P}$ & $\mathrm{H}$ \\
\hline S. labyrinthiformis (S. G. Gmelin) Gomont & & & & $\mathrm{C}$ & & & & \\
\hline S. subsalsa Oersted ex Gomont & & $\mathrm{L}$ & $\mathrm{F}$ & $\mathrm{C}$ & $\mathrm{T}$ & & & $\mathrm{H}$ \\
\hline S. subtilissima Kützing ex Gomont & & & & $\mathrm{C}$ & & & & \\
\hline S. tenerrima Kützing ex Gomont & & & & $\mathrm{C}$ & $\mathrm{T}$ & & & \\
\hline \multicolumn{9}{|l|}{ Symploca Kützing ex Gomont } \\
\hline S. hydnoides (Harvey) Kützing ex Gomont & & & & $\mathrm{C}$ & $\mathrm{T}$ & & & \\
\hline \multicolumn{9}{|l|}{ Oscillatoriaceae (S. F. Gray) Harvey ex Kirchaer } \\
\hline \multicolumn{9}{|l|}{ Blennothrix Kützing ex Gomont } \\
\hline B. lyngbyacea (Kützing ex Gomont) Anagnostidis et Komárek & & $\mathrm{L}$ & $\mathrm{F}$ & $\mathrm{C}$ & $\mathrm{T}$ & & & $\mathrm{H}$ \\
\hline \multicolumn{9}{|l|}{ Lyngbya C. Agardh ex Gomont } \\
\hline L. aestuarii Liebman ex Gomont & & & & $\mathrm{C}$ & $\mathrm{T}$ & & & \\
\hline L. confervoides C. Agardh ex Gomont & & $\mathrm{L}$ & & $\mathrm{C}$ & $\mathrm{T}$ & & & \\
\hline L. lutea (C. Agardh) Gomont & & $\mathrm{L}$ & & $\mathrm{C}$ & $\mathrm{T}$ & & & \\
\hline L. majuscula (Dillwyn) Harvey ex Gomont & & & & $\mathrm{C}$ & $\mathrm{T}$ & & & \\
\hline \multicolumn{9}{|l|}{ Oscillatoria Vaucher ex Gomont } \\
\hline O. margaritifera Kützing ex Gomont & & & & $\mathrm{C}$ & $\mathrm{T}$ & & & \\
\hline O. princeps Vaucher ex Gomont & & $\mathrm{L}$ & & & & & & \\
\hline \multicolumn{9}{|l|}{ Homoeotrichaceae Elenkin } \\
\hline \multicolumn{9}{|l|}{ Heteroleibleinia (Geitler) L. Hoffmann } \\
\hline H. infixa (Frémy) Anagnostidis et Komárek & & & & $\mathrm{C}$ & $\mathrm{T}$ & & & \\
\hline \multicolumn{9}{|l|}{ Nostocales } \\
\hline \multicolumn{9}{|l|}{ Microchaetaceae Lemmermann } \\
\hline \multicolumn{9}{|l|}{ Microchaete Thuret ex Bornet et Flahault } \\
\hline M. vitiensis Askenasy ex Bornet et Flahault & & & & $\mathrm{C}$ & & & & \\
\hline \multicolumn{9}{|l|}{ Nostocaceae Eichler } \\
\hline \multicolumn{9}{|l|}{ Anabaena Bory de Saint-Vincent ex Bornet et Flahault } \\
\hline A. oscillarioides Bory de Saint-Vincent ex Bornet et Flault & & $\mathrm{L}$ & $\mathrm{F}$ & $\mathrm{C}$ & $\mathrm{T}$ & & & \\
\hline A. torulosa (Carmichael) Lagerheim ex Bornet et Flahault & & $\mathrm{L}$ & & $\mathrm{C}$ & & & & \\
\hline \multicolumn{9}{|l|}{ Trichormus (Bornet et Flahault) Komárek et Anagnostidis } \\
\hline T. variabilis (Kützing ex Bornet et Flahault) Komárek et Anagnostidis & & $\mathrm{L}$ & & & & & & \\
\hline \multicolumn{9}{|l|}{ Rivulariaceae (Kützing) Kirchner } \\
\hline \multicolumn{9}{|l|}{ Calothrix C. Agardh ex Bornet et Flahault } \\
\hline C. aeruginea (Kützing) Thuret ex Bornet et Flahault & & $\mathrm{L}$ & & $\mathrm{C}$ & $\mathrm{T}$ & & & \\
\hline C. confervicola (Dillwyn) C. Agardh ex Bornet et Flahault & & $\mathrm{L}$ & & $\mathrm{C}$ & $\mathrm{T}$ & G & & \\
\hline C. consociata (Kützing) Bornet et Flahault & & & & & $\mathrm{T}$ & & & \\
\hline C. crustacea Schousboe et Thuret ex Bornet et Flahault & & $\mathrm{L}$ & $\mathrm{F}$ & $\mathrm{C}$ & $\mathrm{T}$ & $\mathrm{G}$ & $\mathrm{P}$ & $\mathrm{H}$ \\
\hline C. fusco-violacea P. L. Crouan et H. M. Crouan ex Bornet et Flahault & & & & & $\mathrm{T}$ & & & \\
\hline C. scopulorum (Weber et Mohr) C. Agardh ex Bornet et Flahault & & & & $\mathrm{C}$ & & $\mathrm{G}$ & & \\
\hline \multicolumn{9}{|l|}{ Dichothrix Zanardini ex Bornet et Flahault } \\
\hline D. bornetiana Howe & & & & & & & & $\mathrm{H}$ \\
\hline \multicolumn{9}{|l|}{ Isactis Thuret ex Bornet et Flahault } \\
\hline I. plana (Harvey) Thuret ex Bornet et Flahault & & & & $\mathrm{C}$ & & & & \\
\hline Rivularia C. Agardh ex Bornet et Flahault & & & & & & & & \\
\hline R. atra Roth ex Bornet et Flahault & & & & $\mathrm{C}$ & $\mathrm{T}$ & & & \\
\hline R. bullata (Poiret) Berkeley ex Bornet et Flahault & & $\mathrm{L}$ & $\mathrm{F}$ & $\mathrm{C}$ & $\mathrm{T}$ & $\mathrm{G}$ & & $\mathrm{H}$ \\
\hline Scytonemataceae (Kützing) Kirchaer & & & & & & & & \\
\hline Scytonema C. Agardh ex Bornet et Flahault & & & & & & & & \\
\hline S. hofmannii C. Agardh ex Bornet et Flahault & & $\mathrm{L}$ & $\mathrm{F}$ & & $\mathrm{T}$ & & & $\mathrm{H}$ \\
\hline Stigonematales & & & & & & & & \\
\hline Mastigocladaceae Geitler & & & & & & & & \\
\hline Brachytrichia Zanardini ex Bornet et Flahault & & & & & & & & \\
\hline B. quoyi (C. Agardh) Bornet et Flahault & & $\mathrm{L}$ & $\mathrm{F}$ & $\mathrm{C}$ & $\mathrm{T}$ & G & $\mathrm{P}$ & $\mathrm{H}$ \\
\hline Nostochopsidaceae Geitler & & & & & & & & \\
\hline Mastigocoleus Lagerheim ex Bornet et Flahault & & & & & & & & \\
\hline M. testarum Lagerheim ex Bornet et Flahault & & $\mathrm{L}$ & & & & & & \\
\hline
\end{tabular}


Table I. Continued.

\begin{tabular}{|c|c|c|c|c|c|c|c|c|}
\hline & + & $\mathrm{L}$ & $\mathrm{F}$ & $\mathrm{C}$ & $\mathrm{T}$ & $\mathrm{G}$ & $\mathrm{P}$ & $\mathrm{H}$ \\
\hline \multicolumn{9}{|l|}{ RHODOPHYCOTA } \\
\hline \multicolumn{9}{|l|}{ RHODOPHYCEAE } \\
\hline \multicolumn{9}{|l|}{ BANGIOPHYCIDAE } \\
\hline \multicolumn{9}{|l|}{ Porphyridiales } \\
\hline \multicolumn{9}{|l|}{ Porphyridiacae Kylin ex Skuja } \\
\hline \multicolumn{9}{|l|}{ Chroodactylon Hansgirg } \\
\hline C. ornatum (C. Agardh) Basson & & $\mathrm{L}$ & & $\mathrm{C}$ & $\mathrm{T}$ & & & \\
\hline \multicolumn{9}{|l|}{ Rhodosorus Geitler } \\
\hline R. marinus Geitler & & & & $\mathrm{C}$ & & & & \\
\hline \multicolumn{9}{|l|}{ Stylonema Reinsch } \\
\hline S. alsidii (Zanardini) K. M. Drew & & $\mathrm{L}$ & $\mathrm{F}$ & $\mathrm{C}$ & $\mathrm{T}$ & & & $\mathrm{H}$ \\
\hline S. cornu-cervi Reinsch & & & & & $\mathrm{T}$ & & & \\
\hline \multicolumn{9}{|l|}{ Compsogonales } \\
\hline \multicolumn{9}{|l|}{ Erythrotrichiaceae G. M. Smith } \\
\hline \multicolumn{9}{|l|}{ Erythrocladia Rosenvinge } \\
\hline E. irregularis Rosenvinge & + & & & & & & & \\
\hline \multicolumn{9}{|l|}{ Erythrotrichia Areschoug } \\
\hline E. carnea (Dillwyn) J. Agardh & & & $\mathrm{F}$ & $\mathrm{C}$ & $\mathrm{T}$ & & & $\mathrm{H}$ \\
\hline \multicolumn{9}{|l|}{ Porphyrostromium Trévisan de Saint-Léon } \\
\hline P. boryanum (Montagne) P. Silva & & & & $\mathrm{C}$ & & & & \\
\hline P. ciliare (Carmichael ex Harvey) M. J. Wynne & & & & $\mathrm{C}$ & & & & \\
\hline \multicolumn{9}{|l|}{ Sahlingia Kornmann } \\
\hline S. subintegra (Rosenvinge) Kornmann & & & & & & & & $\mathrm{H}$ \\
\hline \multicolumn{9}{|l|}{ Bangiales } \\
\hline \multicolumn{9}{|l|}{ Bangiaceae Nägeli } \\
\hline \multicolumn{9}{|l|}{ Bangia Lyngbye } \\
\hline B. atropurpurea (Roth) C. Agardh & & $\mathrm{L}$ & $\mathrm{F}$ & $\mathrm{C}$ & $\mathrm{T}$ & G & & \\
\hline \multicolumn{9}{|l|}{ Porphyra C. Agardh } \\
\hline P. carolinensis Coll et $\mathrm{J}$. Cox (5) & & $\mathrm{L}$ & $\mathrm{F}$ & $\mathrm{C}$ & $\mathrm{T}$ & & & \\
\hline P. leucosticta Thuret & & & & $\mathrm{C}$ & $\mathrm{T}$ & & & \\
\hline P. umbilicalis (Linnaeus) J. Agardh & & & & $\mathrm{C}$ & & & & \\
\hline \multicolumn{9}{|l|}{ FLORIDEOPHYCIDAE } \\
\hline \multicolumn{9}{|l|}{ Acrochaetiales } \\
\hline Acrochaetiaceae Fritsch ex W. R. Taylor & & & & & & & & \\
\hline Acrochaetium Nägeli & & & & & & & & \\
\hline A. barbadensis (Vickers) Børgesen & & & & $\mathrm{C}$ & $\mathrm{T}$ & & & \\
\hline A. canariense Børgesen & & $\mathrm{L}$ & & $\mathrm{C}$ & $\mathrm{T}$ & & & \\
\hline A. crassipes (Børgesen) Børgesen & & & & & $\mathrm{T}$ & & & \\
\hline A. cymopoliae $\mathrm{B} \varnothing$ rgesen & & & & $\mathrm{C}$ & & & & \\
\hline A. daviesii (Dillwyn) Nägeli & & $\mathrm{L}$ & $\mathrm{F}$ & & $\mathrm{T}$ & $\mathrm{G}$ & $\mathrm{P}$ & \\
\hline A. gracile $\mathrm{B} \emptyset$ rgesen & & $\mathrm{L}$ & & $\mathrm{C}$ & & & $\mathrm{P}$ & \\
\hline A. infestans M. Howe et Hoyt & & & & & $\mathrm{T}$ & & & \\
\hline A. liagorae $\mathrm{B} \emptyset$ rgesen & & & & & $\mathrm{T}$ & & & \\
\hline A. microscopicum (Nägeli ex Kützing) Nägeli & & $\mathrm{L}$ & & $\mathrm{C}$ & $\mathrm{T}$ & & & \\
\hline A. nemalionis (De Notaris ex L. Dufour) Bornet & & & & $\mathrm{C}$ & & & & \\
\hline A. parvulum (Kylin) Hoyt & & $\mathrm{L}$ & & $\mathrm{C}$ & & & & \\
\hline A. savianum (Meneghini) Nägeli & & & & $\mathrm{C}$ & & & & \\
\hline A. secundatum (Lyngbye) Nägeli & & $\mathrm{L}$ & & $\mathrm{C}$ & & & & \\
\hline
\end{tabular}

\section{Palmariales}

Rhodothamniellaceae G. W. Saunders

Rhodothamniella Feldmann

$R$. codicola (Børgesen) Bidoux et Magne

$\begin{array}{llllll}\text { L } & \text { F } & \text { C } & \text { T } & \text { G } & \text { P }\end{array}$

\section{Nemaliales}

Galaxauraceae P. G. Parkinson

Galaxaura J. V. Lamouroux

G. obtusata (J. Ellis et Solander) J. V. Lamouroux

G. rugosa (J. Ellis et Solander) J. V. Lamouroux

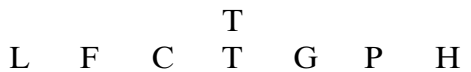


Table I. Continued.

\begin{tabular}{|c|c|c|c|c|c|c|c|c|}
\hline & + & $\mathrm{L}$ & $\mathrm{F}$ & $\mathrm{C}$ & $\mathrm{T}$ & G & $\mathrm{P}$ & $\mathrm{H}$ \\
\hline \multicolumn{9}{|l|}{ Scinaia Bivona-Bernardi } \\
\hline S. australis (Setchell) Huisman (6) & & & & & $\mathrm{T}$ & & & \\
\hline S. caribaea (W. R. Taylor) Huisman (6) & & & $\mathrm{F}$ & & $\mathrm{T}$ & & & \\
\hline S. complanata (Collins) Cotton & & $\mathrm{L}$ & $\mathrm{F}$ & $\mathrm{C}$ & $\mathrm{T}$ & & & \\
\hline S. furcellata (Turner) J. Agardh & & $\mathrm{L}$ & $\mathrm{F}$ & $\mathrm{C}$ & $\mathrm{T}$ & $\mathrm{G}$ & & \\
\hline \multicolumn{9}{|l|}{ Tricleocarpa Huisman et Borowitzka } \\
\hline T. cylindrica (J. Ellis et Solander) Huisman et Borowitzka & & $\mathrm{L}$ & $\mathrm{F}$ & $\mathrm{C}$ & $\mathrm{T}$ & & $\mathrm{P}$ & \\
\hline T. fragilis (Linnaeus) Huisman et Townsend & & $\mathrm{L}$ & $\mathrm{F}$ & & $\mathrm{T}$ & $\mathrm{G}$ & $\mathrm{P}$ & $\mathrm{H}$ \\
\hline \multicolumn{9}{|l|}{ Liagoraceae Kützing } \\
\hline \multicolumn{9}{|l|}{ Ganonema K. C. Fan et Y. C. Wang } \\
\hline G. farinosa (J. V. Lamouroux) K. C. Fan et Y. C. Wang & & & $\mathrm{F}$ & $\mathrm{C}$ & $\mathrm{T}$ & & & $\mathrm{H}$ \\
\hline G. lubrica Afonso-Carrillo, Sansón et Reyes & & & & $\mathrm{C}$ & $\mathrm{T}$ & & $\mathrm{P}$ & $\mathrm{H}$ \\
\hline \multicolumn{9}{|l|}{ Helminthocladia J. Agardh } \\
\hline H. agardhiana P.S. Dixon & & & & & $\mathrm{T}$ & & & \\
\hline H. calvadosii (J. V. Lamouroux ex Duby) Setchell & & $\mathrm{L}$ & & & $\mathrm{T}$ & & & $\mathrm{H}$ \\
\hline \multicolumn{9}{|l|}{ Helminthora J. Agardh } \\
\hline H. stackhousei (Clemente) Cremandes et Pérez-Cirera & + & & & & & & & \\
\hline \multicolumn{9}{|l|}{ Liagora J. V. Lamouroux } \\
\hline L. albicans J. V. Lamouroux & & & & $\mathrm{C}$ & $\mathrm{T}$ & & & \\
\hline L. canariensis Børgesen & & $\mathrm{L}$ & $\mathrm{F}$ & $\mathrm{C}$ & $\mathrm{T}$ & $\mathrm{G}$ & $\mathrm{P}$ & $\mathrm{H}$ \\
\hline L. ceranoides J. V. Lamouroux & & $\mathrm{L}$ & & $\mathrm{C}$ & $\mathrm{T}$ & & & \\
\hline L. distenta (Mertens ex Roth) J. V. Lamouroux & & $\mathrm{L}$ & $\mathrm{F}$ & $\mathrm{C}$ & $\mathrm{T}$ & G & $\mathrm{P}$ & \\
\hline L. gymnarthron $\mathrm{B} \emptyset$ rgesen & & & & $\mathrm{C}$ & & & & \\
\hline L. maderensis Kützing & & & & & & & & $\mathrm{H}$ \\
\hline L. tetrasporifera $\mathrm{B} \emptyset$ rgesen & & $\mathrm{L}$ & $\mathrm{F}$ & $\mathrm{C}$ & $\mathrm{T}$ & & $\mathrm{P}$ & $\mathrm{H}$ \\
\hline L. valida Harvey (7) & & & $\mathrm{F}$ & $\mathrm{C}$ & $\mathrm{T}$ & & & $\mathrm{H}$ \\
\hline L. viscida (Forsskål) C. Agardh & & $\mathrm{L}$ & & & $\mathrm{T}$ & & & \\
\hline \multicolumn{9}{|l|}{ Nemalion Duby } \\
\hline N. helminthoides (Velley) Batters & & $\mathrm{L}$ & & $\mathrm{C}$ & $\mathrm{T}$ & G & $\mathrm{P}$ & \\
\hline \multicolumn{9}{|l|}{ Trichogloeopsis I. A. Abbott et Doty } \\
\hline T. pedicellata (Howe) I. A. Abbott et Doty & & & & & & & $\mathrm{P}$ & $\mathrm{H}$ \\
\hline
\end{tabular}

\section{Gelidiales}

Gelidiaceae Kützing

Gelidium J. V. Lamouroux

G. arbuscula Bory de Saint-Vincent ex Børgesen

G. canariense (Grunow) Seoane-Camba ex Haroun, Gil-Rodríguez, Díaz

de Castro et Prud'homme van Reine (8)

G. crinale (Turner) Gaillon (9)

G. microdon Kützing (10)

G. pusillum (Stackhouse) Le Jolis

G. spathulatum (Kützing) Bornet

G. spinosum (S. G. Gmelin) P. Silva

Pterocladiella Santelices et Hommersand

P. capillacea (S. G. Gmelin) Santelices et Hommersand

P. melanoidea (Schousboe ex Bornet) Santelices et Hommersand

Gelidiellaceae K. C. Fan

Gelidiella Feldmann et G. Hamel

G. antipai Celan

G. pannosa (Feldmann) Feldmann et G. Hamel

G. tinerfensis Seoane-Camba

$\begin{array}{ccccccc} & & \mathrm{C} & \mathrm{T} & \mathrm{G} & \mathrm{P} & \\ & & \mathrm{C} & \mathrm{T} & \mathrm{G} & \mathrm{P} & \\ & & & & & \\ & & \mathrm{C} & & & & \\ & & \mathrm{C} & \mathrm{T} & & & \\ \mathrm{L} & \mathrm{F} & \mathrm{C} & \mathrm{T} & \mathrm{G} & \mathrm{P} & \mathrm{H} \\ & & & \mathrm{T} & & & \\ & & & & & & \mathrm{H}\end{array}$

$\begin{array}{llllllll}\text { L } & \text { F } & \text { C } & \text { T } & \text { G } & \text { P } & \text { H }\end{array}$

$\begin{array}{ll} & \mathrm{T} \\ \mathrm{L} & \mathrm{T}\end{array}$

\section{Gracilariales}

Gracilariaceae Nägeli

Gracilaria Greville

G. armata (C. Agardh) Greville

G. cervicornis (Turner) J. Agardh (11)

G. confervoides (Linnaeus) Greville (12)

G. dura (C. Agardh) J. Agardh (13)

G. longa Gargiulo, De Masi et Tripodi

G. multipartita (Clemente) Harvey

$\begin{array}{ccccc} & & \mathrm{C} & \\ & & \mathrm{C} & \\ \mathrm{L} & \mathrm{F} & & \mathrm{T} \\ \mathrm{L} & & \mathrm{C} & \\ & & & \mathrm{T} \\ \mathrm{L} & & & \mathrm{T}\end{array}$


Table I. Continued.

\begin{tabular}{|c|c|c|c|c|c|c|c|c|}
\hline & + & $\mathrm{L}$ & $\mathrm{F}$ & $\mathrm{C}$ & $\mathrm{T}$ & G & $\mathrm{P}$ & $\mathrm{H}$ \\
\hline G. verrucosa (Hudson) Papenfuss (12) & & $\mathrm{L}$ & & $\mathrm{C}$ & & & & \\
\hline \multicolumn{9}{|l|}{ Pterocladiophilaceae K. C. Fan et Papenfuss } \\
\hline \multicolumn{9}{|l|}{ Gelidiocolax N. L. Gardner } \\
\hline G. microsphaericus N. L. Gardner & & & & & & & & $\mathrm{H}$ \\
\hline
\end{tabular}

\section{Bonnemaisoniales}

Bonnemaisoniaceae F. Schmitz

Asparagopsis Montagne

A. armata Harvey (14)

A. taxiformis (Delile) Trevisan de Saint-Léon (14)

Bonnemaisonia C. Agardh

B. hamifera Hariot (15)

Naccariaceae Kylin

Naccaria Endlicher

$N$. wigghii (Turner) Endlicher

\section{Cryptonemiales}

Acrosymphytaceae S. C. Lindstrom

Acrosymphyton G. Sjöstedt

A. purpuriferum (J. Agardh) G. Sjöstedt

Dumontiaceae Bory de Saint-Vincent

Dudresnaya P. L. Crouan et H. M. Crouan

D. canariensis Tabares, Afonso-Carrillo, Sansón et Reyes

D. crassa Howe (16)

D. verticillata (Withering) Le Jolis

Gloiosiphoniaceae F. Schmitz

Atractophora H. L. Crouan et P. M. Crouan

A. hypnoides P. L. Crouan et H. M. Crouan (17)

Schimmelmannia Schousboe ex Kützing

S. schousboei (J. Agardh) J. Agardh (18)

Thuretella F. Schmitz

T. schousboei (Thuret) F. Schmitz

Halymeniaceae Bory de Saint-Vincent

Cryptonemia J. Agardh

C. crenulata (J. Agardh) J. Agardh

C. lomation (Bertoloni) J. Agardh

C. seminervis (C. Agardh) J. Agardh

Grateloupia C. Agardh

G. dichotoma J. Agardh

G. doryphora (Montagne) M. Howe

G. filicina (J. V. Lamouroux) C. Agardh

Halymenia C. Agardh

H. elongata $\mathrm{C}$. Agardh

H. floresia (Clemente y Rubio) C. Agardh

H. floridana J. Agardh

H. latifolia P. L. Crouan et H. M. Crouan ex Kützing

H. rosea $\mathrm{M}$. Howe et $\mathrm{W}$. R. Taylor

H. vinacea $\mathrm{M}$. Howe et W. R. Taylor

Kallymeniaceae W. R. Taylor

Kallymenia J. Agardh

K. feldmannii Codomier

K. reniformis (Turner) J. Agardh

$K$. requienii J. Agardh

Meredithia J. Agardh

M. microphylla (J. Agardh) J. Agardh

Peyssonneliaceae Denizot

Peyssonnelia Decaisne

P. armorica (P.L Crouan et H. M. Crouan) Weber-van Bosse

P. dubyi PL. Crouan et H. M. Crouan

P. harveyana P. L. Crouan et H. M. Crouan ex J. Agardh

P. inamoena Pilger

P. polymorpha (Zanardini) F. Schmitz
$\begin{array}{lllllll}\mathrm{L} & \mathrm{F} & \mathrm{C} & \mathrm{T} & \mathrm{G} & \mathrm{P} & \mathrm{H}\end{array}$
$\begin{array}{lllllll}\mathrm{L} & \mathrm{F} & \mathrm{C} & \mathrm{T} & \mathrm{G} & \mathrm{P} & \mathrm{H}\end{array}$
$\begin{array}{lllll}\mathrm{L} & \mathrm{F} & \mathrm{C} & \mathrm{T} & \mathrm{H}\end{array}$

F T

F $\quad \mathrm{T}$

$\mathrm{H}$

$\mathrm{T}$

$\begin{array}{llllll}\text { L } & \text { C } & \text { T } & \text { P } & \text { H }\end{array}$

$\mathrm{T}$

$\mathrm{L}$

T $\quad \mathrm{P} \quad \mathrm{H}$

$\begin{array}{llll}\mathrm{L} & \mathrm{F} & \mathrm{T}\end{array}$

$\begin{array}{llll}\mathrm{L} & \mathrm{F} & \mathrm{C} & \mathrm{T} \\ \mathrm{L} & \mathrm{F} & & \end{array}$

L $\quad$ C T

C $\mathrm{T}$

$\mathrm{T}$

$\begin{array}{llll} & \mathrm{F} & & \mathrm{T} \\ \mathrm{L} & \mathrm{F} & \mathrm{C} & \\ \mathrm{F} & & \mathrm{T}\end{array}$

L F C

L $\quad \mathrm{F}$

L $\quad \mathrm{F} \quad \mathrm{T}$

$\mathrm{P}$

$\begin{array}{llll}\mathrm{L} & \mathrm{F} & & \\ & \mathrm{F} & \mathrm{C} & \mathrm{T}\end{array}$

$\begin{array}{llll} & \mathrm{F} & \mathrm{C} & \mathrm{T} \\ & \mathrm{F} & \mathrm{T}\end{array}$

$\begin{array}{llll}\mathrm{L} & \mathrm{F} & \mathrm{C} & \mathrm{T}\end{array}$

F $\quad \mathrm{T}$

L $\mathrm{F}$

$\begin{array}{llll}\mathrm{L} & \mathrm{F} & \mathrm{C} & \mathrm{T}\end{array}$ 
Table I. Continued.

\begin{tabular}{|c|c|c|c|c|c|c|c|c|}
\hline & + & $\mathrm{L}$ & $\mathrm{F}$ & $\mathrm{C}$ & $\mathrm{T}$ & G & $\mathrm{P}$ & $\mathrm{H}$ \\
\hline P. rubra (Greville) J. Agardh & & $\mathrm{L}$ & & $\mathrm{C}$ & $\mathrm{T}$ & & & \\
\hline $\begin{array}{l}\text { Hildenbrandiales } \\
\text { Hildenbrandiaceae Rosenvinge }\end{array}$ & & & & & & & & \\
\hline $\begin{array}{l}\text { Hildenbrandia Nardo } \\
\text { H. crouanii J. Agardh } \\
\text { H. occidentalis Setchell } \\
\text { H. rubra (Sommerfelt) Meneghini }\end{array}$ & & & & $\mathrm{C}$ & $\begin{array}{l}\mathrm{T} \\
\mathrm{T} \\
\mathrm{T}\end{array}$ & & & \\
\hline
\end{tabular}

\section{Corallinales}

Corallinaceae J. V. Lamouroux

Amphiroa J. V. Lamouroux

A. beauvoisii J. V. Lamouroux

A. fragilissima (Linnaeus) J. V. Lamouroux

A. rigida J. V. Lamouroux

Choreonema F. Schmitz

C. thuretii (Bornet) F. Schmitz

Corallina Linnaeus

C. elongata J. Ellis et Solander

C. officinalis Linnaeus

Fosliella M. Howe

F. paschalis (Me. Lemoine) Setchell et N. L. Gardner

Haliptilon (Montagne ex Kützing) Lindley

$H$. cubense (Montagne ex Kützing) Garbary et $\mathrm{H}$. W. Johansen

H. squamatum (Linnaeus) H. W. Johansen, L. M. Irvine et A. M. Webster + (19)

H. virgatum (Zanardini) Garbary et $\mathrm{H}$. W. Johansen

Hydrolithon (Foslie) Foslie

H. boreale (Foslie) Y. M. Chamberlain

H. cruciatum (Bressan) Y. M. Chamberlain

H. farinosum ( J. V. Lamouroux) Penrose et Y. M. Chamberlain

H. onkodes (Heydrich) Penrose et Woelkerling

H. samoënse (Foslie) Keats et Y. M. Chamberlain

Jania J. V. Lamouroux

J. adhaerens J. V. Lamouroux

J. capillacea Harvey

J. longifurca Zanardini

J. pumila J. V. Lamouroux

J. rubens (Linnaeus) J. V. Lamouroux

Lithophyllum Philippi

L. cystoseirae (Hauck) Heydrich

L. esperi Heydrich

L. incrustans Philippi

L. irregulare (Foslie) Huvé ex Steentoft

L. lobatum Me. Lemoine

L. vickersiae Me. Lemoine

Lithoporella (Foslie) Foslie

L. sauvageaui (Foslie) W. H. Adey

Lithothamnion Heydrich

L. corallioides (P. L. Crouan et H. M. Crouan) P. L. Crouan et H. M. Crouan

L. sonderi Hauck

Melobesia J. V. Lamouroux

M. membranacea (Esper) J. V. Lamouroux

Mesophyllum Me. Lemoine

M. canariense (Foslie) Me. Lemoine

M. ectocarpon (Foslie) W. H. Adey

M. lichenoides (J. Ellis) Me. Lemoine

Neogoniolithon Setchell et L. R. Mason

$N$. accretum (Foslie et Howe) Setchell et L. R. Mason

N. caribaeum (Foslie) W. H. Adey

$\mathrm{L} \quad \mathrm{C} \quad \mathrm{T} \quad \mathrm{H}$

$\begin{array}{llll}\mathrm{L} & \mathrm{F} & \mathrm{C} & \mathrm{T} \\ \mathrm{L} & & \mathrm{C} & \mathrm{T}\end{array}$

$\mathrm{H}$

$\begin{array}{llll}\text { L } & \text { F } & \text { T }\end{array}$

$\begin{array}{lllllll}\text { L } & \text { F } & \text { C } & \text { T } & \text { G } & \text { P } & \text { H }\end{array}$

$\begin{array}{llllll}\mathrm{L} & \mathrm{F} & \mathrm{T} & \mathrm{G} & \mathrm{H}\end{array}$

N. hirtum (Me. Lemoine) Afonso-Carrillo

N. orotavicum (Foslie) Me. Lemoine

Phymatolithon Foslie

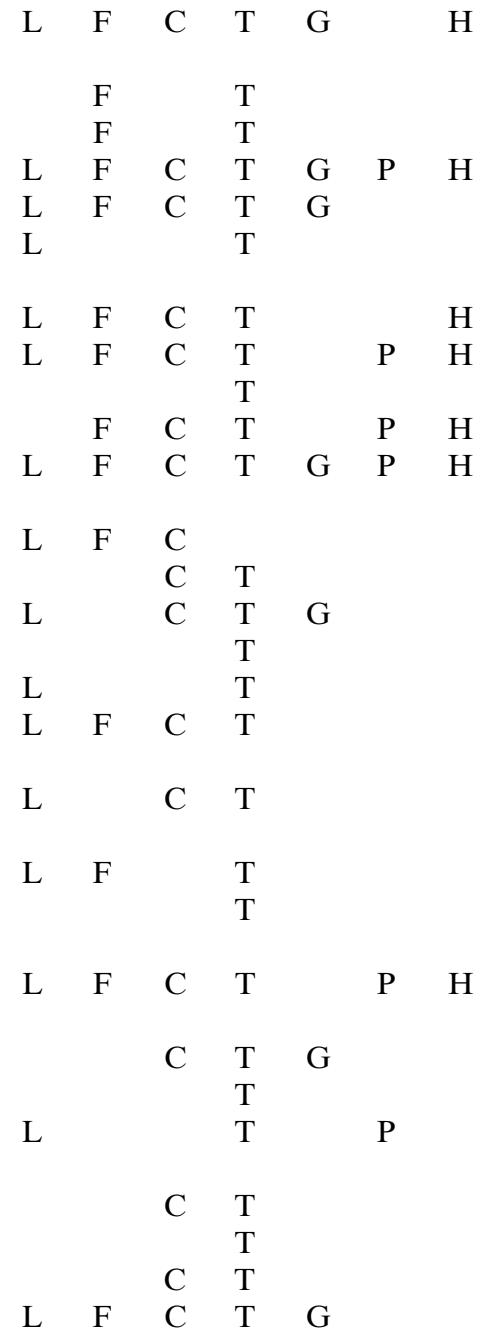


Table I. Continued.

\begin{tabular}{|c|c|c|c|c|c|c|c|c|}
\hline & + & $\mathrm{L}$ & $\mathrm{F}$ & $\mathrm{C}$ & $\mathrm{T}$ & G & $\mathrm{P}$ & $\mathrm{H}$ \\
\hline P. bisporum (Foslie) Afonso-Carrillo & & $\mathrm{L}$ & & $\mathrm{C}$ & $\mathrm{T}$ & $\mathrm{G}$ & & \\
\hline P. calcareum (Pallas) W. H. Adey et D. L. McKibbin & & $\mathrm{L}$ & & $\mathrm{C}$ & $\mathrm{T}$ & & & \\
\hline P. lenormandii (Areschoug) W. H. Adey & & $\mathrm{L}$ & & $\mathrm{C}$ & $\mathrm{T}$ & & & $\mathrm{H}$ \\
\hline P. tenuissimum (Foslie) W.H. Adey & & & & $\mathrm{C}$ & $\mathrm{T}$ & & & \\
\hline \multicolumn{9}{|l|}{ Pneophyllum Kützing } \\
\hline P. confervicolum (Kützing) Y. M. Chamberlain & & $\mathrm{L}$ & & & & & & \\
\hline$P$ fragile Kützing & & $\mathrm{L}$ & $\mathrm{F}$ & $\mathrm{C}$ & $\mathrm{T}$ & & & $\mathrm{H}$ \\
\hline \multicolumn{9}{|l|}{ Schmitziella Bornet et Batters } \\
\hline S. endophloea Bornet et Batters & & & & & $\mathrm{T}$ & & & \\
\hline \multicolumn{9}{|l|}{ Titanoderma Nägeli } \\
\hline $\begin{array}{l}\text { T. corallinae (P. L. Crouan et H. M. Crouan) Woelkerling, Y. M. Cham- } \\
\text { berlain } \text { et } \text { P. Silva }\end{array}$ & & $\mathrm{L}$ & & $\mathrm{C}$ & & & & \\
\hline T. polycephalum (Foslie) Woelkerling, Y. M. Chamberlain et P. Silva & & & & & $\mathrm{T}$ & & & $\mathrm{H}$ \\
\hline T. pustulatum (J. V. Lamouroux) Nägeli & & $\mathrm{L}$ & & $\mathrm{C}$ & $\mathrm{T}$ & & & \\
\hline \multicolumn{9}{|l|}{ Sporolithaceae Verheij } \\
\hline \multicolumn{9}{|l|}{ Sporolithon Heydrich } \\
\hline S. africanum (Foslie) Afonso-Carrillo & & & & & $\mathrm{T}$ & & & \\
\hline \multicolumn{9}{|l|}{ Gigartinales } \\
\hline \multicolumn{9}{|l|}{ Calosiphoniaceae Kylin } \\
\hline \multicolumn{9}{|l|}{ Calosiphonia P. L. Crouan et H. M. Crouan } \\
\hline C. vermicularis (J. Agardh) F. Schmitz & + & & & & & & & \\
\hline \multicolumn{9}{|l|}{ Caulacanthaceae Kützing } \\
\hline \multicolumn{9}{|l|}{ Catenella Greville } \\
\hline C. caespitosa (Withering) L. M. Irvine & & $\mathrm{L}$ & & & $\mathrm{T}$ & & & \\
\hline \multicolumn{9}{|l|}{ Caulacanthus Kützing } \\
\hline C. ustulatus (Mertens ex Turner) Kützing & & $\mathrm{L}$ & & $\mathrm{C}$ & $\mathrm{T}$ & $\mathrm{G}$ & $\mathrm{P}$ & $\mathrm{H}$ \\
\hline \multicolumn{9}{|l|}{ Feldmannophycus $\mathrm{H}$. Augier et C. Boudouresque } \\
\hline F. rayssiae (J. et G. Feldmann) H. Augier et C. Boudouresque & & & & & & & & $\mathrm{H}$ \\
\hline \multicolumn{9}{|l|}{ Cystocloniaceae Kützing } \\
\hline \multicolumn{9}{|l|}{ Rhodophyllis Kützing } \\
\hline$R$. divaricata (Stackhouse) Papenfuss & & $\mathrm{L}$ & & & $\mathrm{T}$ & & & \\
\hline \multicolumn{9}{|l|}{ Furcellariaceae Greville } \\
\hline \multicolumn{9}{|l|}{ Halarachnion Kützing } \\
\hline H. ligulatum (Woodward) Kützing & & $\mathrm{L}$ & $\mathrm{F}$ & & $\mathrm{T}$ & & $\mathrm{P}$ & \\
\hline \multicolumn{9}{|l|}{ Gigartinaceae Bory de Saint-Vincent } \\
\hline \multicolumn{9}{|l|}{ Chondracanthus Kützing } \\
\hline C. acicularis (Roth) Fredericq & & & $\mathrm{F}$ & $\mathrm{C}$ & $\mathrm{T}$ & $\mathrm{G}$ & $\mathrm{P}$ & \\
\hline \multicolumn{9}{|l|}{ Gigartina Stackhouse } \\
\hline G. pistillata (S. G. Gmelin) Stackhouse & & & & & $\mathrm{T}$ & G & $\mathrm{P}$ & \\
\hline \multicolumn{9}{|l|}{ Hypneaceae J. Agardh } \\
\hline \multicolumn{9}{|l|}{ Hypnea J. V. Lamouroux } \\
\hline H. arbuscula P. Dangeard & & & $\mathrm{F}$ & & & & & \\
\hline H. flagelliformis (Greville) J. Agardh & + & & & & & & & \\
\hline H. musciformis (Wulfen) J. V. Lamouroux & & $\mathrm{L}$ & $\mathrm{F}$ & $\mathrm{C}$ & $\mathrm{T}$ & $\mathrm{G}$ & $\mathrm{P}$ & $\mathrm{H}$ \\
\hline H. spinella (C. Agardh) Kützing & & $\mathrm{L}$ & $\mathrm{F}$ & $\mathrm{C}$ & $\mathrm{T}$ & & & $\mathrm{H}$ \\
\hline H. valentiae (Turner) Montagne & & & & & $\mathrm{T}$ & & & \\
\hline Hypneocolax $\mathrm{B} \varnothing$ rgesen & & & & & & & & \\
\hline H. stellaris Børgesen (20) & & & & & $\mathrm{T}$ & & & \\
\hline
\end{tabular}

Nemastomataceae F. Schmitz

Itonoa Masuda et Guiry

I. marginifera (J. Agardh) Masuda et Guiry

Nemastoma J. Agardh

$N$. canariense (Kützing) Montagne

Predaea G. De Toni

P. huismanii Kraft

P. masonii (Setchell et N. L. Gardner) G. de Toni

P. ollivieri Feldmann

L F

P. pusilla (Berthold) Feldmann

Tsengia K. C. Fan et Y. P. Fan

T. bairdii (Farlow) K. C. Fan et Y. P. Fan

Petrocelidaceae Denizot 
Table I. Continued.

\begin{tabular}{|c|c|c|c|c|c|c|c|c|}
\hline & + & $\mathrm{L}$ & $\mathrm{F}$ & $\mathrm{C}$ & $\mathrm{T}$ & G & $\mathrm{P}$ & $\mathrm{H}$ \\
\hline \multicolumn{9}{|l|}{ Mastocarpus Kützing } \\
\hline M. stellatus (Stackhouse ex Whitering) Guiry & & & $\mathrm{F}$ & & $\mathrm{T}$ & & & \\
\hline \multicolumn{9}{|l|}{ Phyllophoraceae Rabenhorst } \\
\hline \multicolumn{9}{|l|}{ Gymnogongrus Martius } \\
\hline G. crenulatus (Turner) J. Agardh & & $\mathrm{L}$ & & & $\mathrm{T}$ & & & \\
\hline G. griffithsiae (Turner) Martius & & & & $\mathrm{C}$ & $\mathrm{T}$ & & & $\mathrm{H}$ \\
\hline \multicolumn{9}{|l|}{ Phyllophora Greville } \\
\hline P. crispa (Hudson) P. S. Dixon & & & & & $\mathrm{T}$ & & & \\
\hline P. gelidioides P. L. Crouan et H. M. Crouan ex Karsakoff & & & $\mathrm{F}$ & $\mathrm{C}$ & & & $\mathrm{P}$ & \\
\hline \multicolumn{9}{|l|}{ Stenogramme Harvey } \\
\hline S. interrupta (C. Agardh) Montagne ex Harvey & & $\mathrm{L}$ & $\mathrm{F}$ & $\mathrm{C}$ & & & & $\mathrm{H}$ \\
\hline \multicolumn{9}{|l|}{ Rissoellaceae Kylin } \\
\hline \multicolumn{9}{|l|}{ Rissoella J. Agardh } \\
\hline R. verruculosa (Bertoloni) J. Agardh & & & & $\mathrm{C}$ & $\mathrm{T}$ & & & \\
\hline \multicolumn{9}{|l|}{ Schizymeniaceae (F. Schmitz et Hauptfleisch) Masuda et Guiry (21) } \\
\hline \multicolumn{9}{|l|}{ Platoma Schousboe ex F. Schmitz } \\
\hline P. cyclocolpum (Montagne) F. Schmitz & & $\mathrm{L}$ & & $\mathrm{C}$ & $\mathrm{T}$ & & & $\mathrm{H}$ \\
\hline \multicolumn{9}{|l|}{ Sebdeniaceae Kylin } \\
\hline \multicolumn{9}{|l|}{ Sebdenia (J. Agardh) Berthold (22) } \\
\hline S. dichotoma Berthold & & & $\mathrm{F}$ & & $\mathrm{T}$ & & & \\
\hline S. macaronesica Soler-Onis, Haroun et Prud'homme van Reine & & & $\mathrm{F}$ & & $\mathrm{T}$ & & & $\mathrm{H}$ \\
\hline S. rodrigueziana (Feldmann) Codomier & & $\mathrm{L}$ & $\mathrm{F}$ & & & & & \\
\hline \multicolumn{9}{|l|}{ Solieriaceae J. Agardh } \\
\hline \multicolumn{9}{|l|}{ Meristotheca J. Agardh } \\
\hline M. decumbens Grunow & & $\mathrm{L}$ & & & $\mathrm{T}$ & & & \\
\hline \multicolumn{9}{|l|}{ Sarcodiotheca Kylin } \\
\hline S. divaricata W. R. Taylor & & & $\mathrm{F}$ & & & & & \\
\hline \multicolumn{9}{|l|}{ Solieria J. Agardh } \\
\hline S. filiformis (Kützing) P. W. Gabrielson & & & & $\mathrm{C}$ & & & & \\
\hline \multicolumn{9}{|l|}{ Sphaerococcaceae Dumortier } \\
\hline \multicolumn{9}{|l|}{ Sphaerococcus Stackhouse } \\
\hline S. coronopifolius (Goodenough et Woodward) Stackhouse & & & & $\mathrm{C}$ & $\mathrm{T}$ & & & \\
\hline \multicolumn{9}{|l|}{ Wurdemanniaceae W. R. Taylor } \\
\hline \multicolumn{9}{|l|}{ Wurdemannia Harvey } \\
\hline W. miniata (Sprengel) Feldmann et Hamel & & $\mathrm{L}$ & & $\mathrm{C}$ & $\mathrm{T}$ & & & \\
\hline \multicolumn{9}{|l|}{ Plocamiales } \\
\hline \multicolumn{9}{|l|}{ Plocamiaceae Kützing } \\
\hline \multicolumn{9}{|l|}{ Plocamium J. V. Lamouroux } \\
\hline P. cartilagineum (Linnaeus) P. S. Dixon & & $\mathrm{L}$ & $\mathrm{F}$ & $\mathrm{C}$ & $\mathrm{T}$ & G & & \\
\hline
\end{tabular}

Rhodymeniales

Champiaceae Kützing

Champia Desvaux

C. parvula (C. Agardh) Harvey

C. salicornioides Harvey

C. vieillardii Kützing

Chylocladia Greville ex Hooker

C. verticillata (Lightfoot) Bliding

Gastroclonium Kützing

G. clavatum (Roth) Ardissone

G. reflexum (Chauvin) Kützing

Lomentariaceae J. Agardh

Lomentaria Lyngbye
L. articulata (Hudson) Lyngbye
L. baileyana (Harvey) Farlow
L. linearis Zanardini
L. subdichotoma Ercegovic
$\begin{array}{lllllll}\mathrm{L} & \mathrm{F} & \mathrm{C} & \mathrm{T} & \mathrm{G} & \mathrm{P} & \mathrm{H}\end{array}$
F
$\mathrm{T}$
F C
$\begin{array}{llllll}\mathrm{L} & \mathrm{F} & \mathrm{C} & \mathrm{T} & \mathrm{G}\end{array}$
$\mathrm{L}$
$\mathrm{T}$

Rhodymeniaceae Harvey

Asteromenia Huisman et A. Millar

A. peltata (W. R. Taylor) Huisman et A. Millar

Botryocladia (J. Agardh) Kylin

$\begin{array}{lllllll}\text { L } & \text { F } & \text { C } & \text { T } & \text { G } & \text { P } & \text { H } \\ \text { L } & & & & & & \\ \text { L } & \text { F } & \text { C } & & & & \\ \text { L } & & & & & & \end{array}$ 
Table I. Continued.

\begin{tabular}{|c|c|c|c|c|c|c|c|c|}
\hline & + & $\mathrm{L}$ & $\mathrm{F}$ & $\mathrm{C}$ & $\mathrm{T}$ & G & $\mathrm{P}$ & $\mathrm{H}$ \\
\hline B. boergesenii Feldmann & & $\mathrm{L}$ & $\mathrm{F}$ & & $\mathrm{T}$ & & & \\
\hline B. botryoides (Wulfen) Feldmann & & $\mathrm{L}$ & $\mathrm{F}$ & $\mathrm{C}$ & $\mathrm{T}$ & $\mathrm{G}$ & $\mathrm{P}$ & $\mathrm{H}$ \\
\hline B. chiajeana (Meneghini) Kylin & & $\mathrm{L}$ & $\mathrm{F}$ & $\mathrm{C}$ & $\mathrm{T}$ & & $\mathrm{P}$ & \\
\hline B. pyriformis (Børgesen) Kylin & & $\mathrm{L}$ & $\mathrm{F}$ & $\mathrm{C}$ & $\mathrm{T}$ & & & \\
\hline B. wynnei D. L. Ballantine & & & $\mathrm{F}$ & & & & $\mathrm{P}$ & \\
\hline \multicolumn{9}{|l|}{ Chrysymenia J. Agardh } \\
\hline C. enteromorpha Harvey & & $\mathrm{L}$ & $\mathrm{F}$ & & & & & \\
\hline C. ventricosa (J. V. Lamouroux) J. Agardh & & & $\mathrm{F}$ & & & & & \\
\hline \multicolumn{9}{|l|}{ Coelarthrum Børgesen } \\
\hline C. cliftonii (Harvey) Kylin & & $\mathrm{L}$ & & & & & & \\
\hline \multicolumn{9}{|l|}{ Coelothrix Børgesen } \\
\hline C. irregularis (Harvey) Børgesen & & $\mathrm{L}$ & & & $\mathrm{T}$ & & & \\
\hline \multicolumn{9}{|l|}{ Fauchea Montagne et Bory de Saint-Vicent } \\
\hline F. repens (C. Agardh) Montagne et Bory de Saint-Vicent & & $\mathrm{L}$ & & & & & & \\
\hline \multicolumn{9}{|l|}{ Gelidiopsis F. Schmitz } \\
\hline G. intricata (C. Agardh) Vickers & & $\mathrm{L}$ & & $\mathrm{C}$ & $\mathrm{T}$ & G & & $\mathrm{H}$ \\
\hline G. planicaulis (W. R. Taylor) W. R. Taylor & & & & $\mathrm{C}$ & & & & \\
\hline \multicolumn{9}{|l|}{ Gloiocladia J. Agardh } \\
\hline G. atlantica (Searles) R. E. Norris & & & & $\mathrm{C}$ & & & & \\
\hline G. blomquistii (Searles) R. E. Norris & & & $\mathrm{F}$ & & & & & \\
\hline \multicolumn{9}{|l|}{ Halichrysis (J. Agardh) F. Schmitz } \\
\hline H. depressa (J. Agardh) F. Schmitz & & & & & $\mathrm{T}$ & & & \\
\hline \multicolumn{9}{|l|}{ Leptofauchea Kylin } \\
\hline L. brasiliensis A. B. Joly & & & & & $\mathrm{T}$ & & & \\
\hline \multicolumn{9}{|l|}{ Rhodymenia Greville } \\
\hline$R$. ardissonei Feldmann & & $\mathrm{L}$ & $\mathrm{F}$ & & $\mathrm{T}$ & & & \\
\hline R. caespitosa P. A. Dangeard & & $\mathrm{L}$ & $\mathrm{F}$ & $\mathrm{C}$ & & & & \\
\hline R. holmesii Ardissone & & $\mathrm{L}$ & & $\mathrm{C}$ & & & & \\
\hline R. pseudopalmata (J. V. Lamouroux) P. Silva & & $\mathrm{L}$ & $\mathrm{F}$ & $\mathrm{C}$ & $\mathrm{T}$ & G & $\mathrm{P}$ & $\mathrm{H}$ \\
\hline
\end{tabular}

\section{Ceramiales}

Ceramiaceae Dumortier

Aglaothamnion Feldmann-Mazoyer

A. byssoides (Arnott ex Harvey) L'Hardy-Halos et Rueness

A. cordatum (Børgesen) Feldmann-Mazoyer

A. gallicum (Nägeli) L'Hardy-Halos et Ardré

A. hookeri (Dillwyn) Maggs et Hommersand

A. neglectum Feldmann-Mazoyer

A. tenuissimum (Bonnemaison) Feldmann-Mazoyer

Anotrichium Nägeli

A. barbatum (C. Agardh) Nägeli

A. furcellatum (J. Agardh) Baldock

A. tenue (C. Agardh) Nägeli

Antithamnion Nägeli

A. cruciatum (C. Agardh) Nägeli

A. decipiens (J. Agardh) Athanasiadis (23)

A. densum (Suhr) Howe

A. diminuatum E. M. Wollaston

A. lherminieri (P. L. Crouan et H. M. Crouan) Bornet ex Nasr

A. ogdeniae Abbott

Antithamnionella Lyle

A. boergesenii (Cormaci et Furnari) Athanasiadis (24)

A. elegans (Berthold) J. H. Price et D. M. John

Bornetia Thuret

B. secundiflora (J. Agardh) Thuret

$\begin{array}{llllll}\mathrm{L} & & \mathrm{C} & \mathrm{T} & \mathrm{G} & \mathrm{P} \\ \mathrm{L} & \mathrm{F} & \mathrm{C} & \mathrm{T} & & \\ & & \mathrm{C} & \mathrm{T} & & \end{array}$

$\mathrm{L}$

$\begin{array}{llll} & \mathrm{C} & & \\ \mathrm{L} & \mathrm{C} & \mathrm{T} & \mathrm{G}\end{array}$

$\begin{array}{lllll}\mathrm{L} & \mathrm{F} & \mathrm{C} & \mathrm{T} & \mathrm{H}\end{array}$

$\begin{array}{lllllll}\mathrm{L} & \mathrm{F} & \mathrm{C} & \mathrm{T} & \mathrm{G} & \mathrm{H}\end{array}$

$\begin{array}{lllll}\mathrm{L} & \mathrm{F} & \mathrm{C} & \mathrm{T} & \mathrm{H}\end{array}$

$\begin{array}{lllllll}\mathrm{L} & \mathrm{F} & \mathrm{C} & \mathrm{T} & \mathrm{G} & \mathrm{P} & \mathrm{H}\end{array}$

$+$

L $\quad \mathrm{F} \quad \mathrm{C}$

L $\quad$ F $\quad$ C $\quad$ T

$\begin{array}{lllllll} & \text { F } & \text { C } & \text { T } & \text { G } & \\ \text { L } & \text { F } & \text { C } & & & & \text { H }\end{array}$

$\begin{array}{llllll}\text { L } & \text { F } & \text { C } & \text { T } & \text { G } & \text { P }\end{array}$

Callithamniella Feldmann-Mazoyer

C. tingitana (Schousboe ex Bornet) Feldmann-Mazoyer

Callithamnion Lyngbye

C. corymbosum (Smith) Lyngbye

C. ellipticum Montagne

C. granulatum (Ducluzeau) C. Agardh

C. tetragonum (Withering) Gray 
Table I. Continued.

\begin{tabular}{|c|c|c|c|c|c|c|c|c|}
\hline & + & $\mathrm{L}$ & $\mathrm{F}$ & $\mathrm{C}$ & $\mathrm{T}$ & G & $\mathrm{P}$ & $\mathrm{H}$ \\
\hline \multicolumn{9}{|l|}{ Centroceras Kützing } \\
\hline C. clavulatum (C. Agardh) Montagne & & $\mathrm{L}$ & $\mathrm{F}$ & $\mathrm{C}$ & $\mathrm{T}$ & G & $\mathrm{P}$ & $\mathrm{H}$ \\
\hline \multicolumn{9}{|l|}{ Centrocerocolax A. B. Joly } \\
\hline C. ubatubensis A. B. Joly & & $\mathrm{L}$ & $\mathrm{F}$ & $\mathrm{C}$ & $\mathrm{T}$ & & & \\
\hline \multicolumn{9}{|l|}{ Ceramium Roth } \\
\hline C. atrorubescens Kylin & & & $\mathrm{F}$ & & $\mathrm{T}$ & & & \\
\hline C. ciliatum (J. Ellis) Ducluzeau & & $\mathrm{L}$ & $\mathrm{F}$ & $\mathrm{C}$ & $\mathrm{T}$ & G & $\mathrm{P}$ & $\mathrm{H}$ \\
\hline C. cingulatum Weber-van Bosse & & & $\mathrm{F}$ & & $\mathrm{T}$ & & & \\
\hline C. circinatum (Kützing) J. Agardh & & $\mathrm{L}$ & $\mathrm{F}$ & $\mathrm{C}$ & $\mathrm{T}$ & & $\mathrm{P}$ & $\mathrm{H}$ \\
\hline C. codii (H. Richards) Feldmann-Mazoyer & & $\mathrm{L}$ & $\mathrm{F}$ & $\mathrm{C}$ & $\mathrm{T}$ & & & $\mathrm{H}$ \\
\hline C. deslongchampii Chauvin ex Duby & & & & $\mathrm{C}$ & $\mathrm{T}$ & & & \\
\hline C. diaphanum (Lightfoot) Roth & & $\mathrm{L}$ & $\mathrm{F}$ & $\mathrm{C}$ & $\mathrm{T}$ & G & $\mathrm{P}$ & $\mathrm{H}$ \\
\hline C. echionotum J. Agardh & & $\mathrm{L}$ & $\mathrm{F}$ & $\mathrm{C}$ & $\mathrm{T}$ & $\mathrm{G}$ & $\mathrm{P}$ & $\mathrm{H}$ \\
\hline C. flaccidum (Kützing) Ardissone & & $\mathrm{L}$ & $\mathrm{F}$ & $\mathrm{C}$ & $\mathrm{T}$ & & $\mathrm{P}$ & $\mathrm{H}$ \\
\hline C. gaditanum (Clemente y Rubio) Cremades & & $\mathrm{L}$ & & $\mathrm{C}$ & $\mathrm{T}$ & & $\mathrm{P}$ & \\
\hline C. rubrum C. Agardh & & $\mathrm{L}$ & $\mathrm{F}$ & $\mathrm{C}$ & $\mathrm{T}$ & $\mathrm{G}$ & $\mathrm{P}$ & $\mathrm{H}$ \\
\hline C. tenerrimum (Martens) Okamura & & $\mathrm{L}$ & $\mathrm{F}$ & $\mathrm{C}$ & $\mathrm{T}$ & & & \\
\hline \multicolumn{9}{|l|}{ Compsothamnion Nägeli } \\
\hline C. decompositum (J. Agardh) Maggs et L'Hardy-Halos & & & & & $\mathrm{T}$ & & & \\
\hline C. thuyoides (Smith) Nägeli & & & & $\mathrm{C}$ & $\mathrm{T}$ & & & \\
\hline \multicolumn{9}{|l|}{ Crouania J. Agardh } \\
\hline C. attenuata (C. Agardh) J. Agardh & & $\mathrm{L}$ & $\mathrm{F}$ & $\mathrm{C}$ & $\mathrm{T}$ & $\mathrm{G}$ & $\mathrm{P}$ & $\mathrm{H}$ \\
\hline \multicolumn{9}{|l|}{ Diplothamnion A. B. Joly et Yamagushi-Tomita } \\
\hline D. jolyi C. van den Hoek & & $\mathrm{L}$ & & & & & $\mathrm{P}$ & \\
\hline \multicolumn{9}{|l|}{ Grallatoria M. Howe } \\
\hline G. reptans M. Howe & & & & & $\mathrm{T}$ & & & \\
\hline \multicolumn{9}{|l|}{ Griffithsia J. Agardh } \\
\hline G. capitata $\mathrm{B} \emptyset$ rgesen & & & & $\mathrm{C}$ & $\mathrm{T}$ & & & \\
\hline G. opuntioides J. Agardh & & $\mathrm{L}$ & $\mathrm{F}$ & $\mathrm{C}$ & $\mathrm{T}$ & & $\mathrm{P}$ & \\
\hline G. phyllamphora J. Agardh & & $\mathrm{L}$ & $\mathrm{F}$ & $\mathrm{C}$ & $\mathrm{T}$ & G & $\mathrm{P}$ & \\
\hline G. radicans Kützing & & & $\mathrm{F}$ & & & & & \\
\hline G. schousboei Montagne & & & & $\mathrm{C}$ & $\mathrm{T}$ & & & \\
\hline \multicolumn{9}{|l|}{ Gymnophycus Huisman et Kraft } \\
\hline G. hapsiphorus Huisman et Kraft & & $\mathrm{L}$ & $\mathrm{F}$ & & $\mathrm{T}$ & & & $\mathrm{H}$ \\
\hline \multicolumn{9}{|l|}{ Gymnothamnion J. Agardh } \\
\hline G. elegans (Schousboe ex C. Agardh) J. Agardh & & $\mathrm{L}$ & $\mathrm{F}$ & $\mathrm{C}$ & $\mathrm{T}$ & $\mathrm{G}$ & $\mathrm{P}$ & \\
\hline \multicolumn{9}{|l|}{ Halurus Kützing } \\
\hline H. equisetifolius (Lightfoot) Kützing & & $\mathrm{L}$ & $\mathrm{F}$ & & & & & \\
\hline \multicolumn{9}{|l|}{ Lejolisia Bornet } \\
\hline L. mediterranea Bornet & & & & $\mathrm{C}$ & & & & \\
\hline \multicolumn{9}{|l|}{ Monosporus Solier } \\
\hline M. pedicellatus (Smith) Solier & & & $\mathrm{F}$ & $\mathrm{C}$ & $\mathrm{T}$ & & & \\
\hline \multicolumn{9}{|l|}{ Pleonosporium Nägeli } \\
\hline P. borreri (Smith) Nägeli & & $\mathrm{L}$ & & $\mathrm{C}$ & $\mathrm{T}$ & & & \\
\hline P. caribaeum (Børgesen) R. E. Norris & & & $\mathrm{F}$ & $\mathrm{C}$ & $\mathrm{T}$ & & & $\mathrm{H}$ \\
\hline Pterothamnion Nägeli & & & & & & & & \\
\hline P. crispum (Ducluzeau) Nägeli & & & $\mathrm{F}$ & & & & & \\
\hline P. plumula (J. Ellis) Nägeli & & $\mathrm{L}$ & $\mathrm{F}$ & $\mathrm{C}$ & & & & \\
\hline Ptilothamnion Thuret & & & & & & & & \\
\hline P. pluma (Dillwyn) Thuret & & $\mathrm{L}$ & & & & & & \\
\hline P. speluncarum (Collins et Hervey) Ballantine et M. J. Wynne & & & $\mathrm{F}$ & & $\mathrm{T}$ & & & \\
\hline Seirospora Harvey & & & & & & & & \\
\hline S. interrupta (Smith) F. Schmitz & & & $\mathrm{F}$ & & & & & \\
\hline Spermothamnion Areschoug & & & & & & & & \\
\hline S. flabellatum Bornet & & $\mathrm{L}$ & $\mathrm{F}$ & & & & & \\
\hline S. repens (Dillwyn) Rosenvinge & & $\mathrm{L}$ & $\mathrm{F}$ & $\mathrm{C}$ & $\mathrm{T}$ & & $\mathrm{P}$ & \\
\hline Sphondylothamnion Nägeli & & & & & & & & \\
\hline S. multifidum (Hudson) Nägeli & & $\mathrm{L}$ & $\mathrm{F}$ & & & & & \\
\hline Spyridia Harvey & & & & & & & & \\
\hline S. filamentosa (Wulfen) Harvey & & $\mathrm{L}$ & $\mathrm{F}$ & $\mathrm{C}$ & $\mathrm{T}$ & G & $\mathrm{P}$ & $\mathrm{H}$ \\
\hline S. hypnoides (Bory de Saint-Vincent) Papenfuss & & $\mathrm{L}$ & $\mathrm{F}$ & $\mathrm{C}$ & $\mathrm{T}$ & & $\mathrm{P}$ & $\mathrm{H}$ \\
\hline
\end{tabular}


Table I. Continued.

\begin{tabular}{|c|c|c|c|c|c|c|c|c|}
\hline & + & $\mathrm{L}$ & $\mathrm{F}$ & $\mathrm{C}$ & $\mathrm{T}$ & G & $\mathrm{P}$ & $\mathrm{H}$ \\
\hline \multicolumn{9}{|l|}{ Tiffaniella Doty et Meñez } \\
\hline T. capitata (Schousboe ex Bornet) Doty et Meñez & & $\mathrm{L}$ & $\mathrm{F}$ & $\mathrm{C}$ & & & & \\
\hline T. gorgonea (Montagne) Doty et Meñez & & $\mathrm{L}$ & & $\mathrm{C}$ & $\mathrm{T}$ & & & \\
\hline \multicolumn{9}{|l|}{ Vickersia Karsakoff } \\
\hline V. baccata (J. Agardh) Karsakoff & & $\mathrm{L}$ & $\mathrm{F}$ & $\mathrm{C}$ & $\mathrm{T}$ & $\mathrm{G}$ & $\mathrm{P}$ & $\mathrm{H}$ \\
\hline \multicolumn{9}{|l|}{ Wrangelia C. Agardh } \\
\hline W. argus (Montagne) Montagne & & & $\mathrm{F}$ & $\mathrm{C}$ & $\mathrm{T}$ & & $\mathrm{P}$ & $\mathrm{H}$ \\
\hline W. penicillata (C. Agardh) C. Agardh & & $\mathrm{L}$ & $\mathrm{F}$ & $\mathrm{C}$ & $\mathrm{T}$ & & & $\mathrm{H}$ \\
\hline \multicolumn{9}{|l|}{ Dasyaceae Kützing } \\
\hline \multicolumn{9}{|l|}{ Dasya C. Agardh } \\
\hline D. baillouviana (S. G. Gmelin) Montagne & & $\mathrm{L}$ & & $\mathrm{C}$ & $\mathrm{T}$ & & $\mathrm{P}$ & $\mathrm{H}$ \\
\hline D. caraibica $\mathrm{B} \emptyset$ rgesen & & & & & $\mathrm{T}$ & & & \\
\hline D. corymbifera J. Agardh & & $\mathrm{L}$ & $\mathrm{F}$ & $\mathrm{C}$ & $\mathrm{T}$ & & & $\mathrm{H}$ \\
\hline D. crouaniana J. Agardh & & & & & $\mathrm{T}$ & & & \\
\hline D. hutchinsiae Harvey & & $\mathrm{L}$ & $\mathrm{F}$ & $\mathrm{C}$ & $\mathrm{T}$ & & & $\mathrm{H}$ \\
\hline D. ocellata (Grateloup) Harvey & & $\mathrm{L}$ & $\mathrm{F}$ & $\mathrm{C}$ & $\mathrm{T}$ & $\mathrm{G}$ & $\mathrm{P}$ & $\mathrm{H}$ \\
\hline D. rigidula (Kützing) Ardissone & & $\mathrm{L}$ & $\mathrm{F}$ & & $\mathrm{T}$ & & & \\
\hline \multicolumn{9}{|l|}{ Eupogodon Kützing } \\
\hline E. planus (J. Agardh) Kützing & & $\mathrm{L}$ & $\mathrm{F}$ & & & & & \\
\hline E. spinellus (C. Agardh) Kützing & & $\mathrm{L}$ & & & & & & \\
\hline \multicolumn{9}{|l|}{ Halydictyon Zanardini } \\
\hline H. mirabile Zanardini & & $\mathrm{L}$ & $\mathrm{F}$ & $\mathrm{C}$ & $\mathrm{T}$ & & & $\mathrm{H}$ \\
\hline \multicolumn{9}{|l|}{ Heterosiphonia Montagne } \\
\hline H. crispella (C. Agardh) M. J. Wynne & & $\mathrm{L}$ & $\mathrm{F}$ & $\mathrm{C}$ & $\mathrm{T}$ & $\mathrm{G}$ & $\mathrm{P}$ & $\mathrm{H}$ \\
\hline \multicolumn{9}{|l|}{ Delesseriaceae Bory de Saint-Vincent } \\
\hline \multicolumn{9}{|l|}{ Acrosorium Zanardini ex Kützing } \\
\hline \multirow{2}{*}{\multicolumn{9}{|c|}{$\begin{array}{l}\text { A. venulosum (Lanardmi) Kyпn } \\
\text { Apoglossum J. Agardh }\end{array}$}} \\
\hline & & & & & & & & \\
\hline A. ruscifolium (Turner) J. Agardh & & $\mathrm{L}$ & $\mathrm{F}$ & & $\mathrm{T}$ & & & \\
\hline \multicolumn{9}{|l|}{ Branchioglossum Kylin } \\
\hline B. prostratum C. W. Schneider & & & $\mathrm{F}$ & & & & & \\
\hline \multicolumn{9}{|l|}{ Cottoniella Børgesen } \\
\hline C. filamentosa (M. Howe) Børgesen & & $\mathrm{L}$ & $\mathrm{F}$ & $\mathrm{C}$ & $\mathrm{T}$ & $\mathrm{G}$ & $\mathrm{P}$ & $\mathrm{H}$ \\
\hline \multicolumn{9}{|l|}{ Cryptopleura Kützing } \\
\hline C. ramosa (Hudson) Kylin ex L. Newton & & $\mathrm{L}$ & $\mathrm{F}$ & & & & & \\
\hline Drachiella J. Ernst et Feldmann & & & & & & & & \\
\hline D. minuta (Kylin) Maggs et Hommersand & & $\mathrm{L}$ & & & $\mathrm{T}$ & & & \\
\hline Haraldia Feldmann & & & & & & & & \\
\hline H. lenormandii (Derbès et Solier) Feldmann & & $\mathrm{L}$ & & & & & & \\
\hline Hypoglossum Kützing & & & & & & & & \\
\hline H. hypoglossoides (Stackhouse) Collins et Hervey & & $\mathrm{L}$ & $\mathrm{F}$ & $\mathrm{C}$ & $\mathrm{T}$ & G & $\mathrm{P}$ & \\
\hline Nitophyllum Greville & & & & & & & & \\
\hline N. punctatum (Stackhouse) Greville & & $\mathrm{L}$ & $\mathrm{F}$ & & $\mathrm{T}$ & & & \\
\hline Platysiphonia Børgesen & & & & & & & & \\
\hline P. caribaea D. L. Ballantine et M. J. Wynne & & $\mathrm{L}$ & & & & & & \\
\hline P. delicata (Clemente y Rubio) Cremades & & $\mathrm{L}$ & $\mathrm{F}$ & $\mathrm{C}$ & $\mathrm{T}$ & & & $\mathrm{H}$ \\
\hline Taenioma J. Agardh & & & & & & & & \\
\hline T. nanum (Kützing) Papenfuss & & & $\mathrm{F}$ & & $\mathrm{T}$ & G & & \\
\hline T. perpusillum (J. Agardh) J. Agardh & & $\mathrm{L}$ & $\mathrm{F}$ & $\mathrm{C}$ & $\mathrm{T}$ & & & \\
\hline Rhodomelaceae Areschoug & & & & & & & & \\
\hline Alsidium C. Agardh & & & & & & & & \\
\hline A. corallinum C. Agardh & & $\mathrm{L}$ & $\mathrm{F}$ & $\mathrm{C}$ & & & & \\
\hline Aphanocladia Falkenberg & & & & & & & & \\
\hline A. stichidiosa (Funk) Ardré & & $\mathrm{L}$ & & $\mathrm{C}$ & $\mathrm{T}$ & & & \\
\hline Boergeseniella Kylin & & & & & & & & \\
\hline B. fruticulosa (Wulfen) Kylin & & $\mathrm{L}$ & $\mathrm{F}$ & & $\mathrm{T}$ & & $\mathrm{P}$ & $\mathrm{H}$ \\
\hline Chondria C. Agardh & & & & & & & & \\
\hline C. capillaris (Hudson) M. J. Wynne & & $\mathrm{L}$ & $\mathrm{F}$ & $\mathrm{C}$ & $\mathrm{T}$ & G & $\mathrm{P}$ & $\mathrm{H}$ \\
\hline C. coerulescens (J. Agardh) Falkenberg & & & $\mathrm{F}$ & & $\mathrm{T}$ & $\mathrm{G}$ & & \\
\hline C. dasyphylla (Woodward) C. Agardh & & $\mathrm{L}$ & $\mathrm{F}$ & $\mathrm{C}$ & $\mathrm{T}$ & & & $\mathrm{H}$ \\
\hline C. mairei Feldmann-Mazoyer & & & & & $\mathrm{T}$ & & & \\
\hline
\end{tabular}


Table I. Continued.

\begin{tabular}{|c|c|c|c|c|c|c|c|c|}
\hline & + & $\mathrm{L}$ & $\mathrm{F}$ & $\mathrm{C}$ & $\mathrm{T}$ & G & $\mathrm{P}$ & $\mathrm{H}$ \\
\hline \multicolumn{9}{|l|}{ Chondrophycus (Tokida ex Saito) Garbary et Harper } \\
\hline C. corallopsis (Montagne) K. W. Nam & & & $\mathrm{F}$ & & $\mathrm{T}$ & & & $\mathrm{H}$ \\
\hline C. papillosa (C. Agardh) Garbary et Harper (25) & & $\mathrm{L}$ & $\mathrm{F}$ & & $\mathrm{T}$ & & & \\
\hline C. perforata (Bory de Saint-Vincent) K. W. Nam & & $\mathrm{L}$ & $\mathrm{F}$ & $\mathrm{C}$ & $\mathrm{T}$ & $\mathrm{G}$ & $\mathrm{P}$ & $\mathrm{H}$ \\
\hline C. poiteaui (J. V. Lamouroux) K. W. Nam & & $\mathrm{L}$ & $\mathrm{F}$ & & & & & \\
\hline \multicolumn{9}{|l|}{ Ctenosiphonia Falkenberg } \\
\hline C. hypnoides (Welwitsch ex J. Agardh) Falkenberg & & $\mathrm{L}$ & $\mathrm{F}$ & $\mathrm{C}$ & $\mathrm{T}$ & & $\mathrm{P}$ & $\mathrm{H}$ \\
\hline \multicolumn{9}{|l|}{ Digenea C. Agardh } \\
\hline D. simplex (Wulfen) C. Agardh & & $\mathrm{L}$ & $\mathrm{F}$ & & & & & \\
\hline \multicolumn{9}{|l|}{ Dipterosiphonia F. Schmitz et Falkenberg } \\
\hline D. dendritica (C. Agardh) F. Schmitz & & $\mathrm{L}$ & $\mathrm{F}$ & $\mathrm{C}$ & $\mathrm{T}$ & G & $\mathrm{P}$ & $\mathrm{H}$ \\
\hline D. rigens (Schousboe ex C. Agardh) Falkenberg & & $\mathrm{L}$ & $\mathrm{F}$ & $\mathrm{C}$ & $\mathrm{T}$ & & $\mathrm{P}$ & $\mathrm{H}$ \\
\hline \multicolumn{9}{|l|}{ Erythrocystis J. Agardh } \\
\hline E. montagnei (Derbès et Solier) P. Silva & & $\mathrm{L}$ & $\mathrm{F}$ & $\mathrm{C}$ & $\mathrm{T}$ & G & $\mathrm{P}$ & $\mathrm{H}$ \\
\hline \multicolumn{9}{|l|}{ Halopithys Kützing } \\
\hline H. incurva (Hudson) Batters & & $\mathrm{L}$ & $\mathrm{F}$ & $\mathrm{C}$ & $\mathrm{T}$ & G & & \\
\hline \multicolumn{9}{|l|}{ Herposiphonia Nägeli } \\
\hline H. secunda (C. Agardh) Ambronn & & $\mathrm{L}$ & $\mathrm{F}$ & $\mathrm{C}$ & $\mathrm{T}$ & G & $\mathrm{P}$ & $\mathrm{H}$ \\
\hline \multicolumn{9}{|l|}{ Janczewskia Solms-Laubach } \\
\hline J. verrucaeformis Solms-Laubach & & $\mathrm{L}$ & & & $\mathrm{T}$ & & $\mathrm{P}$ & \\
\hline \multicolumn{9}{|l|}{ Laurencia J. V. Lamouroux } \\
\hline L. brongniartii J. Agardh & & $\mathrm{L}$ & & & & & & $\mathrm{H}$ \\
\hline L. chondrioides Børgesen & & $\mathrm{L}$ & & & & & & \\
\hline L. flexilis Setchell & & & $\mathrm{F}$ & & $\mathrm{T}$ & & & \\
\hline L. glandulifera (Kützing) Kützing & & & & & & & & $\mathrm{H}$ \\
\hline L. intricata J. V. Lamouroux & & & & $\mathrm{C}$ & $\mathrm{T}$ & & & \\
\hline L. majuscula (Harvey) Lucas & & $\mathrm{L}$ & $\mathrm{F}$ & $\mathrm{C}$ & $\mathrm{T}$ & & $\mathrm{P}$ & \\
\hline L. microcladia Kützing & & $\mathrm{L}$ & $\mathrm{F}$ & $\mathrm{C}$ & & & & $\mathrm{H}$ \\
\hline L. minuta Vandermeulen, Garbary et Guiry & & & & & $\mathrm{T}$ & & & \\
\hline L. obtusa (Hudson) J. V. Lamouroux & & $\mathrm{L}$ & $\mathrm{F}$ & $\mathrm{C}$ & $\mathrm{T}$ & G & $\mathrm{P}$ & $\mathrm{H}$ \\
\hline L. tenera Tseng & & & & & $\mathrm{T}$ & & & \\
\hline L. viridis Gil-Rodríguez et Haroun & & $\mathrm{L}$ & $\mathrm{F}$ & $\mathrm{C}$ & $\mathrm{T}$ & & $\mathrm{P}$ & $\mathrm{H}$ \\
\hline \multicolumn{9}{|l|}{ Leptosiphonia Kylin } \\
\hline L. schousboei (Thuret) Kylin & & $\mathrm{L}$ & & & & & & \\
\hline \multicolumn{9}{|l|}{ Lophocladia F. Schmitz } \\
\hline L. trichoclados (Mertens ex C. Agardh) F. Schmitz & & $\mathrm{L}$ & $\mathrm{F}$ & $\mathrm{C}$ & $\mathrm{T}$ & & $\mathrm{P}$ & $\mathrm{H}$ \\
\hline \multicolumn{9}{|l|}{ Lophosiphonia Falkenberg } \\
\hline L. cristata Falkenberg & & $\mathrm{L}$ & & & $\mathrm{T}$ & & $\mathrm{P}$ & $\mathrm{H}$ \\
\hline L. obscura (C. Agardh) Falkenberg & & $\mathrm{L}$ & & & $\mathrm{T}$ & & & $\mathrm{H}$ \\
\hline L. reptabunda (Suhr) Kylin & & $\mathrm{L}$ & $\mathrm{F}$ & $\mathrm{C}$ & $\mathrm{T}$ & G & $\mathrm{P}$ & $\mathrm{H}$ \\
\hline \multicolumn{9}{|l|}{ Neosiphonia M.S. Kim et I. K. Lee } \\
\hline N. sphaerocarpa (Børgesen) M. S. Kim et I. K. Lee & & $\mathrm{L}$ & & $\mathrm{C}$ & $\mathrm{T}$ & & $\mathrm{P}$ & $\mathrm{H}$ \\
\hline \multicolumn{9}{|l|}{ Ophidocladus Falkenberg } \\
\hline O. simpliciusculus (P. L. Crouan et H. M. Crouan) Falkenberg & & & $\mathrm{F}$ & $\mathrm{C}$ & $\mathrm{T}$ & & $\mathrm{P}$ & \\
\hline Osmundaria J. V. Lamouroux & & & & & & & & \\
\hline O. volubilis (Linnaeus) R. E. Norris & & $\mathrm{L}$ & $\mathrm{F}$ & & & & & \\
\hline Osmundea Stackhouse & & & & & & & & \\
\hline O. hybrida (De Candolle) K. W. Nam & & & & $\mathrm{C}$ & $\mathrm{T}$ & G & & $\mathrm{H}$ \\
\hline O. pinnatifida (Hudson) Stackhouse & & $\mathrm{L}$ & $\mathrm{F}$ & $\mathrm{C}$ & $\mathrm{T}$ & G & $\mathrm{P}$ & $\mathrm{H}$ \\
\hline O. truncata (Kützing) K. W. Nam et Maggs & & $\mathrm{L}$ & & & $\mathrm{T}$ & & $\mathrm{P}$ & \\
\hline Polysiphonia Greville & & & & & & & & \\
\hline P. atlantica Kapraun et J. N. Norris & & $\mathrm{L}$ & $\mathrm{F}$ & $\mathrm{C}$ & $\mathrm{T}$ & $\mathrm{G}$ & $\mathrm{P}$ & $\mathrm{H}$ \\
\hline P. breviarticulata (C. Agardh) Zanardini & & & $\mathrm{F}$ & $\mathrm{C}$ & $\mathrm{T}$ & & $\mathrm{P}$ & $\mathrm{H}$ \\
\hline P. brodiaei (Dillwyn) Sprengel & & $\mathrm{L}$ & & & & & & \\
\hline P. carettia Hollenberg & & & & & $\mathrm{T}$ & & & \\
\hline P. ceramiaeformis P. L. Crouan et H. M. Crouan & & & & & $\mathrm{T}$ & & $\mathrm{P}$ & \\
\hline P. denudata (Dillwyn) Greville ex Harvey & & $\mathrm{L}$ & $\mathrm{F}$ & & $\mathrm{T}$ & & $\mathrm{P}$ & $\mathrm{H}$ \\
\hline P. elongata (Hudson) Sprengel & & $\mathrm{L}$ & $\mathrm{F}$ & & $\mathrm{T}$ & $\mathrm{G}$ & & $\mathrm{H}$ \\
\hline P. ferulacea Suhr ex J. Agardh & & $\mathrm{L}$ & $\mathrm{F}$ & $\mathrm{C}$ & $\mathrm{T}$ & G & $\mathrm{P}$ & $\mathrm{H}$ \\
\hline P. fibrillosa (Dillwyn) Sprengel (26) & & $\mathrm{L}$ & $\mathrm{F}$ & $\mathrm{C}$ & $\mathrm{T}$ & G & $\mathrm{P}$ & $\mathrm{H}$ \\
\hline P. flexella (C. Agardh) J. Agardh & & $\mathrm{L}$ & $\mathrm{F}$ & $\mathrm{C}$ & $\mathrm{T}$ & & & \\
\hline
\end{tabular}


Table I. Continued.

\begin{tabular}{|c|c|c|c|c|c|c|c|c|}
\hline & + & $\mathrm{L}$ & $\mathrm{F}$ & $\mathrm{C}$ & $\mathrm{T}$ & $\mathrm{G}$ & $\mathrm{P}$ & $\mathrm{H}$ \\
\hline P. flocculosa (C. Agardh) Kützing & & $\mathrm{L}$ & $\mathrm{F}$ & $\mathrm{C}$ & $\mathrm{T}$ & & $\mathrm{P}$ & \\
\hline P. fucoides (Hudson) Greville & & $\mathrm{L}$ & $\mathrm{F}$ & $\mathrm{C}$ & $\mathrm{T}$ & & & $\mathrm{H}$ \\
\hline P. funebris De Notaris ex J. Agardh & & & & & $\mathrm{T}$ & & $\mathrm{P}$ & \\
\hline P. furcellata (C. Agardh) Harvey & & $\mathrm{L}$ & & $\mathrm{C}$ & $\mathrm{T}$ & & & \\
\hline P. harveyi J. Bailey & & & $\mathrm{F}$ & & $\mathrm{T}$ & & $\mathrm{P}$ & $\mathrm{H}$ \\
\hline P. havanensis Montagne & & & & $\mathrm{C}$ & & & & \\
\hline P. opaca (C. Agardh) Morris et De Notaris & & $\mathrm{L}$ & $\mathrm{F}$ & $\mathrm{C}$ & $\mathrm{T}$ & & $\mathrm{P}$ & $\mathrm{H}$ \\
\hline P. scopulorum Harvey & & $\mathrm{L}$ & $\mathrm{F}$ & & $\mathrm{T}$ & & & $\mathrm{H}$ \\
\hline P. sertularioides (Grateloup) J. Agardh & & $\mathrm{L}$ & $\mathrm{F}$ & $\mathrm{C}$ & $\mathrm{T}$ & $\mathrm{G}$ & $\mathrm{P}$ & $\mathrm{H}$ \\
\hline P. stricta (Dillwyn) Greville & & $\mathrm{L}$ & $\mathrm{F}$ & $\mathrm{C}$ & $\mathrm{T}$ & & & $\mathrm{H}$ \\
\hline P. subulifera (C. Agardh) Harvey & & $\mathrm{L}$ & $\mathrm{F}$ & $\mathrm{C}$ & $\mathrm{T}$ & & $\mathrm{P}$ & $\mathrm{H}$ \\
\hline P. tepida Hollenberg & & $\mathrm{L}$ & $\mathrm{F}$ & $\mathrm{C}$ & & & & $\mathrm{H}$ \\
\hline P. tripinnata J. Agardh & & $\mathrm{L}$ & $\mathrm{F}$ & $\mathrm{C}$ & $\mathrm{T}$ & & & \\
\hline \multicolumn{9}{|l|}{ Pterosiphonia Falkenberg } \\
\hline P. pennata (C. Agardh) Sauvageau & & $\mathrm{L}$ & $\mathrm{F}$ & $\mathrm{C}$ & $\mathrm{T}$ & & & \\
\hline \multicolumn{9}{|l|}{ Rytiphlaea C. Agardh } \\
\hline R. tinctoria (Clemente) C. Agardh & & $\mathrm{L}$ & $\mathrm{F}$ & $\mathrm{C}$ & $\mathrm{T}$ & $\mathrm{G}$ & & \\
\hline \multicolumn{9}{|l|}{ Stichothamnion $\mathrm{B} \emptyset$ rgesen } \\
\hline S. cymatophilum $\mathrm{B} \varnothing$ rgesen & & $\mathrm{L}$ & $\mathrm{F}$ & $\mathrm{C}$ & $\mathrm{T}$ & G & $\mathrm{P}$ & \\
\hline \multicolumn{9}{|l|}{ Womersleyella R. E. Norris } \\
\hline W. setacea (Hollenberg) R. E. Norris (27) & & $\mathrm{L}$ & & $\mathrm{C}$ & $\mathrm{T}$ & $\mathrm{G}$ & $\mathrm{P}$ & $\mathrm{H}$ \\
\hline \multicolumn{9}{|l|}{ Red algae of uncertain systematic position } \\
\hline \multicolumn{9}{|l|}{ Ailocolax Pocock } \\
\hline A. pulchellus Pocock (28) & & & & & $\mathrm{T}$ & & & \\
\hline \multicolumn{9}{|l|}{ CHROMOPHYCOTA } \\
\hline \multicolumn{9}{|l|}{ PHAEOPHYCEAE } \\
\hline \multicolumn{9}{|l|}{ Ectocarpales } \\
\hline \multicolumn{9}{|l|}{ Ectocarpaceae C. Agardh } \\
\hline \multicolumn{9}{|l|}{ Acinetospora Bornet } \\
\hline A. crinita (Carmichael ex Harvey) Kornmann & & & & $\mathrm{C}$ & $\mathrm{T}$ & & & \\
\hline \multicolumn{9}{|l|}{ Asteronema Delépine et Asensi } \\
\hline A. rhodochortonoides (Børgesen) D. G. Müller et E. Parodi & & $\mathrm{L}$ & $\mathrm{F}$ & $\mathrm{C}$ & & & & $\mathrm{H}$ \\
\hline \multicolumn{9}{|l|}{ Ectocarpus Lyngbye } \\
\hline E. fasciculatus Harvey & & $\mathrm{L}$ & & $\mathrm{C}$ & $\mathrm{T}$ & & & \\
\hline E. repens Reinke (29) & & & & $\mathrm{C}$ & $\mathrm{T}$ & & & \\
\hline E. siliculosus (Dillwyn) Lyngbye & & $\mathrm{L}$ & & $\mathrm{C}$ & $\mathrm{T}$ & & $\mathrm{P}$ & \\
\hline \multicolumn{9}{|l|}{ Feldmannia Hamel } \\
\hline F. globifera (Kützing) Hamel & & $\mathrm{L}$ & & $\mathrm{C}$ & $\mathrm{T}$ & $\mathrm{G}$ & $\mathrm{P}$ & \\
\hline F. irregularis (Kützing) Hamel & & $\mathrm{L}$ & $\mathrm{F}$ & $\mathrm{C}$ & $\mathrm{T}$ & & & $\mathrm{H}$ \\
\hline F. paradoxa (Montagne) Hamel & & & & $\mathrm{C}$ & $\mathrm{T}$ & & & \\
\hline Hincksia J. F. Gray & & & & & & & & \\
\hline H. conifera (Børgesen) P. Silva & & & $\mathrm{F}$ & $\mathrm{C}$ & & & & \\
\hline H. intermedia (Rosenvinge) P. Silva & & $\mathrm{L}$ & & $\mathrm{C}$ & & $\mathrm{G}$ & & \\
\hline H. mitchelliae (Harvey) P. Silva & & $\mathrm{L}$ & $\mathrm{F}$ & $\mathrm{C}$ & $\mathrm{T}$ & $\mathrm{G}$ & $\mathrm{P}$ & $\mathrm{H}$ \\
\hline H. onslowensis (Amsler et Kapraun) P. Silva & & $\mathrm{L}$ & $\mathrm{F}$ & & & & & \\
\hline H. rallsiae (Vickers) P. Silva & & $\mathrm{L}$ & & $\mathrm{C}$ & & & & $\mathrm{H}$ \\
\hline H. sandriana (Zanardini) P. Silva & & & & $\mathrm{C}$ & $\mathrm{T}$ & & & \\
\hline Kuckuckia Hamel & & & & & & & & \\
\hline K. spinosa (Kützing) Kuckuck & & $\mathrm{L}$ & & & $\mathrm{T}$ & & & \\
\hline Kuetzingiella Kornmann & & & & & & & & \\
\hline K. battersii (Bornet) Kornmann & & $\mathrm{L}$ & & $\mathrm{C}$ & $\mathrm{T}$ & & & \\
\hline Phaeostroma Kuckuck & & & & & & & & \\
\hline P. pustulosum Kuckuck & & $\mathrm{L}$ & & $\mathrm{C}$ & & & & \\
\hline Pilinia Kützing & & & & & & & & \\
\hline P. rimosa Kützing & & & & $\mathrm{C}$ & $\mathrm{T}$ & & & \\
\hline Spongonema Kützing & & & & & & & & \\
\hline S. tomentosum (Hudson) Kützing & & & & $\mathrm{C}$ & $\mathrm{T}$ & & & \\
\hline Pilayellaceae Pedersen & & & & & & & & \\
\hline Bachelotia (Bornet) Kuckuck ex G. Hamel & & & & & & & & \\
\hline B. antillarum (Grunow) Gerloff & & & & $\mathrm{C}$ & $\mathrm{T}$ & & & \\
\hline
\end{tabular}


Table I. Continued.

\begin{tabular}{|c|c|c|c|c|c|c|c|c|}
\hline & + & $\mathrm{L}$ & $\mathrm{F}$ & $\mathrm{C}$ & $\mathrm{T}$ & $\mathrm{G}$ & $\mathrm{P}$ & $\mathrm{H}$ \\
\hline \multicolumn{9}{|l|}{ Ralfsiaceae Farlow } \\
\hline \multicolumn{9}{|l|}{ Hapalospongidion D. A. Saunders } \\
\hline H. macrocarpum (Feldmann) León-Álvarez et González-González (30) & & & & & $\mathrm{T}$ & & & $\mathrm{H}$ \\
\hline \multicolumn{9}{|l|}{ Nemoderma Schousboe ex Bornet } \\
\hline N. tingitanum Schousboe ex Bornet & & $\mathrm{L}$ & $\mathrm{F}$ & $\mathrm{C}$ & $\mathrm{T}$ & & & $\mathrm{H}$ \\
\hline \multicolumn{9}{|l|}{ Pseudolithoderma Svedelius } \\
\hline P. adriaticum (Hauck) Verlaque & & $\mathrm{L}$ & $\mathrm{F}$ & & $\mathrm{T}$ & & & \\
\hline \multicolumn{9}{|l|}{ Ralfsia Berkeley } \\
\hline R. verrucosa (Areschough) Areschough & & $\mathrm{L}$ & $\mathrm{F}$ & $\mathrm{C}$ & $\mathrm{T}$ & $\mathrm{G}$ & & $\mathrm{H}$ \\
\hline \multicolumn{9}{|l|}{ Sphacelariales } \\
\hline \multicolumn{9}{|l|}{ Choristocarpaceae Kjellman } \\
\hline \multicolumn{9}{|l|}{ Discosporangium Falkenberg } \\
\hline D. mesarthrocarpum (Meneghini) Hauck & & $\mathrm{L}$ & $\mathrm{F}$ & $\mathrm{C}$ & $\mathrm{T}$ & & & \\
\hline \multicolumn{9}{|l|}{ Cladostephaceae Oltmanns } \\
\hline \multicolumn{9}{|l|}{ Cladostephus C. Agardh } \\
\hline C. spongiosus (Hudson) C. Agardh & & $\mathrm{L}$ & $\mathrm{F}$ & $\mathrm{C}$ & $\mathrm{T}$ & & & \\
\hline \multicolumn{9}{|l|}{ Sphacelariaceae Decaisne } \\
\hline \multicolumn{9}{|l|}{ Sphacelaria Lyngbye } \\
\hline S. cirrosa (Roth) C. Agardh & & $\mathrm{L}$ & $\mathrm{F}$ & $\mathrm{C}$ & $\mathrm{T}$ & G & $\mathrm{P}$ & $\mathrm{H}$ \\
\hline S. fusca (Hudson) S. F. Gray & & & $\mathrm{F}$ & & $\mathrm{T}$ & & & \\
\hline S. plumula Zanardini & & $\mathrm{L}$ & & & & & & \\
\hline S. rigidula Kützing & & $\mathrm{L}$ & $\mathrm{F}$ & $\mathrm{C}$ & $\mathrm{T}$ & & & $\mathrm{H}$ \\
\hline S. tribuloides Meneghini & & $\mathrm{L}$ & $\mathrm{F}$ & $\mathrm{C}$ & $\mathrm{T}$ & & & $\mathrm{H}$ \\
\hline \multicolumn{9}{|l|}{ Sphacella Reinke } \\
\hline S. subtilissima Reinke & & & $\mathrm{F}$ & & & & & \\
\hline
\end{tabular}

Stypocaulaceae Oltmanns

Halopteris Kützing

H. filicina (Grateloup) Kützing

Stypocaulon Kützing

S. scoparium (Linnaeus) Kützing

\section{Dictyotales}

Dictyotaceae J. V. Lamouroux ex Dumortier

Dictyopteris J. V. Lamouroux

D. delicatula J. V. Lamouroux

D. plagiogramma (Montagne) Vickers (31)

D. polypodioides (De Candolle) J. V. Lamouroux

Dictyota Lamarck

D. ciliolata Sonder ex Kützing

D. crenulata J. Agardh

D. dichotoma (Hudson) J. V. Lamouroux

D. fasciola (Roth) J. V. Lamouroux

D. humifusa Hörning, Schnetter et Coppejans

D. kohlmeyeri (Nizamuddin et Gerloff) Hörnig, Schnetter et Prud'homme van Reine

D. liturata J. Agardh

D. menstrualis (Hoyt) Schnetter, Hörning et Weber-Peuckert

D. mertensii (Martius) Kützing (32)

D. pfaffii Schnetter (32)

D. pinnatifida Kützing

D. pulchella Hörnig et Schnetter (32)

D. spiralis Montagne

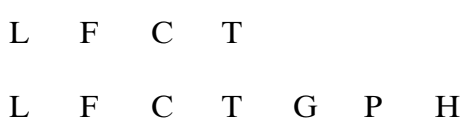

Lobophora J. Agardh

L. variegata (J. V. Lamouroux) Womersley ex E. C. Oliveira

Padina Adanson (33)

P. gymnospora (Kützing) Sonder

P. pavonica (Linnaeus) Thivy

Spatoglossum Kützing

S. schroederi (C. Agardh) Kützing

S. solierii (Chauvin ex Montagne) Kützing

$\begin{array}{lll}\mathrm{L} & \mathrm{T} \\ & \mathrm{C} & \mathrm{T}\end{array}$

$\begin{array}{llllllll}\mathrm{L} & \mathrm{F} & \mathrm{C} & \mathrm{T} & \mathrm{G} & \mathrm{P} & \mathrm{H}\end{array}$

L $\quad$ F $\quad$ C $\quad$ T $\quad$ H

$\begin{array}{llllllllllllllll}\mathrm{L} & \mathrm{F} & \mathrm{T} & \mathrm{P} & \mathrm{H}\end{array}$

$\begin{array}{lllllll}\mathrm{L} & \mathrm{F} & \mathrm{C} & \mathrm{T} & \mathrm{G} & \mathrm{P} & \mathrm{H}\end{array}$

$\begin{array}{lllllll}\mathrm{L} & \mathrm{F} & \mathrm{C} & \mathrm{T} & \mathrm{G} & \mathrm{P} & \mathrm{H}\end{array}$

$\begin{array}{lllllll}\mathrm{L} & \mathrm{F} & \mathrm{C} & \mathrm{T} & \mathrm{G} & \mathrm{P} & \mathrm{H} \\ \mathrm{L} & \mathrm{F} & \mathrm{C} & \mathrm{T} & & \mathrm{P} & \mathrm{H}\end{array}$

Stypopodium Kützing

S. schimperi (Buchinger ex Kützing) Verlaque et C. Boudouresque

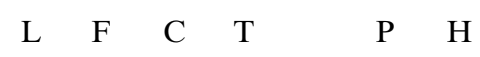

L $\quad \mathrm{F} \quad \mathrm{T}$

$\mathrm{T}$

$\mathrm{L}$

$\begin{array}{lllllll}\text { L } & \text { F } & \text { C } & \text { T } & \text { G } & \text { P } & \text { H }\end{array}$

$\begin{array}{lllllll}\text { L } & \text { F } & \text { C } & \text { T } & \text { G } & \text { P } & \text { H }\end{array}$

$\begin{array}{llllllll}\mathrm{L} & & & \mathrm{T} & & & \\ \mathrm{L} & \mathrm{F} & \mathrm{C} & \mathrm{T} & \mathrm{G} & \mathrm{P} & \mathrm{H}\end{array}$

$\mathrm{T}$

F 
Table I. Continued.

\begin{tabular}{|c|c|c|c|c|c|c|c|c|}
\hline & + & $\mathrm{L}$ & $\mathrm{F}$ & $\mathrm{C}$ & $\mathrm{T}$ & G & $\mathrm{P}$ & $\mathrm{H}$ \\
\hline S. zonale (J. V. Lamouroux) Papenfuss & & $\mathrm{L}$ & $\mathrm{F}$ & $\mathrm{C}$ & $\mathrm{T}$ & G & $\mathrm{P}$ & $\mathrm{H}$ \\
\hline \multicolumn{9}{|l|}{ Taonia J. Agardh } \\
\hline T. atomaria (Woodward) J. Agardh & & $\mathrm{L}$ & $\mathrm{F}$ & $\mathrm{C}$ & $\mathrm{T}$ & $\mathrm{G}$ & $\mathrm{P}$ & $\mathrm{H}$ \\
\hline \multicolumn{9}{|l|}{ Zonaria C. Agardh } \\
\hline Z. tournefortii (J. V. Lamouroux) Montagne & & $\mathrm{L}$ & $\mathrm{F}$ & $\mathrm{C}$ & $\mathrm{T}$ & G & $\mathrm{P}$ & $\mathrm{H}$ \\
\hline
\end{tabular}

\section{Cutleriales}

Cutleriaceae Hauck

Cutleria Greville

C. chilosa (Falkenberg) P. Silva

C. multifida (Smith) Greville

Zanardinia Nardo ex Zanardini

Z. typus (Nardo) G. Furnari

$\begin{array}{lll} & \mathrm{F} & \\ \mathrm{L} & \mathrm{F} & \mathrm{T} \\ & & \mathrm{T}\end{array}$

\section{Chordariales}

Chordariaceae Greville

Cladosiphon Kützing

C. zosterae (J. Agardh) Kylin

Liebmannia J. Agardh

L. leveillei J. Agardh

Mesogloia C. Agardh

M. vermiculata (Smith) S. F. Gray

Papenfussiella Kylin

P. kuromo (Yendo) Inagaki

Sauvageaugloia Hamel ex Kylin

S. chordariaeformis (P. L. Crouan et H. M. Crouan) Kylin

Strepsithalia Bornet ex Sauvageau

S. curvata Sauvageau

Corynophlaeaceae Oltmanns

Corynophlaea Kützing

C. cystophorae J. Agardh

Leathesia S. F. Gray

L. difformis (Linnaeus) Areschoug

Microcoryne Strömfelt

M. ocellata Strömfelt

Myriactula Kuntze

M. chordae (Areschoug) Levring

Petrospongium Nägeli ex Kützing

P. berkeleyi (Greville) Nägeli ex Kützing

Elachistaceae Kjellman

Elachista Duby

E. stellaris (Areschoug) P. Silva

E. globulosa (C. Agardh) J. Agardh

Leptonematella P. Silva

L. fasciculata (Reinke) P. Silva

Myrionemataceae Nägeli

Hecatonema Sauvageau

H. terminale (Kützing) Sauvageau

Myrionema Greville

M. orbiculare J. Agardh

M. strangulans Carmichael ex Greville

Protectocarpus Kornmann

P. speciosus (Børgesen) Kuckuck ex Kornmann

L F

Spermatochnaceae Kjellman

Nemacystus Derbès et Solier

N. erythraeus (J. Agardh) Sauvageau

N. flexuosus (C. Agardh) Kylin

$N$. hispanicus (Sauvageau) Kylin

N. howei (W. R. Taylor) Kylin

F T

$\begin{array}{llll}\mathrm{L} & \mathrm{F} & \mathrm{C} & \mathrm{T}\end{array}$

L

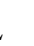

L T

$\mathrm{T}$

Stilophora J. Agardh

S. tenella (Esper) P. Silva

L $\quad$ C T

L C

$\begin{array}{llll}\mathrm{L} & \mathrm{F} & \mathrm{C} & \mathrm{T}\end{array}$

L $\quad \mathrm{C} \quad \mathrm{T}$

C

F T 
Table I. Continued.

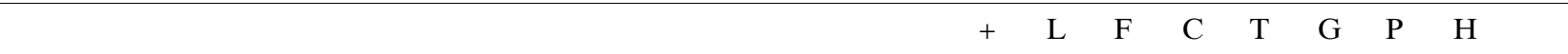

\section{Scytosiphonales}

Scytosiphonaceae Farlow

Colpomenia (Endlicher) Derbès et Solier

C. peregrina (Sauvageau) Hamel (34)

C. sinuosa (Mertens ex Roth) Derbès et Solier

Compsonema Kuckuck

C. microspongium (Batters) Kormann

C. minutum (C. Agardh) Kuckuck

Hydroclathrus Bory de Saint-Vincent

H. clathratus (Bory de Saint-Vicent ex C. Agardh) M. Howe

Petalonia Derbès et Solier

$P$. fascia (O. F. Müller) Kuntze

Rosenvingea $\mathrm{B} \emptyset$ rgesen

R. antillarum (P. L. Crouan et H. M. Crouan) M. J. Wynne

$R$. intricata (J. Agardh) Børgesen

$R$. sanctae-crucis $\mathrm{B} \emptyset$ rgesen

Scytosiphon C. Agardh

S. dotyi M. J. Wynne

S. lomentaria (Lyngbye) Link
L
$\begin{array}{llllllll}\mathrm{L} & \mathrm{F} & \mathrm{C} & \mathrm{T} & \mathrm{G} & \mathrm{P} & \mathrm{H}\end{array}$
L C
$\begin{array}{lllllll}\mathrm{L} & \mathrm{F} & \mathrm{C} & \mathrm{T} & \mathrm{G} & \mathrm{P} & \mathrm{H}\end{array}$
C T

L

L $\quad \mathrm{F} \quad \mathrm{T}$

L $\quad \mathrm{F} \quad \mathrm{T}$

$\mathrm{T}$

$\begin{array}{lllllll}\mathrm{L} & \mathrm{F} & \mathrm{C} & \mathrm{T} & \mathrm{G} & \mathrm{H}\end{array}$

Syringodermatales

Syringodermataceae Henry

Syringoderma Levring

S. floridana Henry

L F

$\mathrm{T}$

Dictyosiphonales

Giraudiaceae Kylin

Giraudia Derbès et Solier

G. sphacelarioides Derbès et Solier

L

$\mathrm{T}$

Myriotrichiaceae Kjellman

Myriotrichia Harvey

M. clavaeformis Harvey

Punctariaceae (Thuret) Kjellmam

Asperococcus J. V. Lamouroux
A. fistulosus (Hudson) Hooker (35)
A. turneri (Smith) Hooker

L F C

\section{Sporochnales}

Sporochnaceae Greville

Carpomitra Kützing

C. costata (Stackhouse) Batters

Nereia Zanardini

N. filiformis (J. Agardh) Zanardini
N. tropica (W. R.Taylor) W. R.Taylor

Sporochnus C. Agardh

S. bolleanus Montagne

S. gaertneri (S. G. Gmelin) C. Agardh

S. pedunculatus (Hudson) C. Agardh
L F
L $\quad \mathrm{F} \quad \mathrm{C} \quad \mathrm{T}$
L $\quad \mathrm{C} \quad \mathrm{T}$
L $\quad \mathrm{F} \quad \mathrm{C} \quad \mathrm{T}$

Desmarestiales

Arthrocladiaceae Chauvin

Arthrocladia Duby

A. villosa (Hudson) Duby

L F

Laminariales

Phyllariaceae Tilden

Saccorhiza Bachelot de la Pylaie

S. polyschides (Lightfoot) Batters

$\mathrm{L}$

\section{Fucales}

Cytoseiraceae de Toni

Cystoseira C. Agardh

C. abies-marina (S. G. Gmelin) C. Agardh

$\begin{array}{llllllll}\text { L } & \text { F } & \text { C } & \text { T } & \text { G } & \text { P } & \text { H }\end{array}$ 
Table I. Continued.

\begin{tabular}{|c|c|c|c|c|c|c|c|c|}
\hline & + & $\mathrm{L}$ & $\mathrm{F}$ & $\mathrm{C}$ & $\mathrm{T}$ & $\mathrm{G}$ & $\mathrm{P}$ & $\mathrm{H}$ \\
\hline C. compressa (Esper) Gerloff et Nizamuddin & & $\mathrm{L}$ & $\mathrm{F}$ & $\mathrm{C}$ & $\mathrm{T}$ & G & $\mathrm{P}$ & $\mathrm{H}$ \\
\hline C. foeniculacea (Linnaeus) Greville & & $\mathrm{L}$ & $\mathrm{F}$ & $\mathrm{C}$ & $\mathrm{T}$ & $\mathrm{G}$ & $\mathrm{P}$ & $\mathrm{H}$ \\
\hline C. humilis Schousboe ex Kützing & & $\mathrm{L}$ & $\mathrm{F}$ & $\mathrm{C}$ & $\mathrm{T}$ & G & $\mathrm{P}$ & $\mathrm{H}$ \\
\hline C. mauritanica Sauvageau & & $\mathrm{L}$ & $\mathrm{F}$ & $\mathrm{C}$ & $\mathrm{T}$ & & & \\
\hline C. tamariscifolia (Hudson) Papenfuss & & $\mathrm{L}$ & $\mathrm{F}$ & $\mathrm{C}$ & & & & \\
\hline C. wildpretii Nizamuddin (36) & & $\mathrm{L}$ & & & & & & \\
\hline \multicolumn{9}{|l|}{ Fucaceae Adanson } \\
\hline \multicolumn{9}{|l|}{ Fucus Linnaeus } \\
\hline F. spiralis Linnaeus & & $\mathrm{L}$ & & $\mathrm{C}$ & $\mathrm{T}$ & & & \\
\hline F. vesiculosus Linnaeus & & $\mathrm{L}$ & & & $\mathrm{T}$ & & & \\
\hline \multicolumn{9}{|l|}{ Sargassaceae Kützing } \\
\hline \multicolumn{9}{|l|}{ Sargassum C. Agardh } \\
\hline S. acinarium (Linnaeus) Setchell & + & & & & & & & \\
\hline S. cymosum C. Agardh & & $\mathrm{L}$ & $\mathrm{F}$ & $\mathrm{C}$ & $\mathrm{T}$ & & & \\
\hline S. desfontainesii (Turner) J. Agardh & & $\mathrm{L}$ & $\mathrm{F}$ & $\mathrm{C}$ & $\mathrm{T}$ & G & $\mathrm{P}$ & $\mathrm{H}$ \\
\hline S. filipendula C. Agardh & & $\mathrm{L}$ & $\mathrm{F}$ & $\mathrm{C}$ & $\mathrm{T}$ & & & \\
\hline S. furcatum Kützing & & & $\mathrm{F}$ & $\mathrm{C}$ & & & & \\
\hline S. natans (Linnaeus) Gaillon & & $\mathrm{L}$ & & & $\mathrm{T}$ & & & \\
\hline S. vulgare C. Agardh & & $\mathrm{L}$ & $\mathrm{F}$ & $\mathrm{C}$ & $\mathrm{T}$ & G & $\mathrm{P}$ & $\mathrm{H}$ \\
\hline \multicolumn{9}{|l|}{ CHLOROPHYCOTA } \\
\hline \multicolumn{9}{|l|}{ Chaetophorales } \\
\hline \multicolumn{9}{|l|}{ Chaetophoraceae Greville } \\
\hline \multicolumn{9}{|l|}{ Acrochaete Pringsheim } \\
\hline A. geniculata (Gardner) O'Kelly (37) & + & & & & & & & \\
\hline A. repens Pringsheim & & $\mathrm{L}$ & & & & & & \\
\hline \multicolumn{9}{|l|}{ Pseudendoclonium Wille } \\
\hline P. fucicola (Rosenvinge) Nielsen & & $\mathrm{L}$ & & & & & & \\
\hline
\end{tabular}

\section{Ctenocladales}

Ulvellaceae Schmidle

Entocladia Reinke

E. viridis Reinke

Epicladia Reinke

E. heterotricha (Yarish) Nielsen

Ochlochaete Twaites

O. hystrix Twaites ex Harvey

Pringsheimiella von Höhnel

P. sanctae-luciae Nielsen et McLachlan

P. scutata (Reinke) von Höhnel ex Marchewianka

Stromatella Kormann et Sahling

S. monostromatica (P. Dangeard) Kornmann et Sahling

Ulvella P. L. Crouan et H. M. Crouan

U. setchellii P. Dangeard

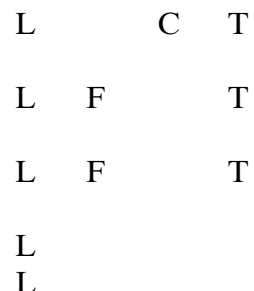

$\mathrm{L}$

F

$\mathrm{T}$

$\mathrm{T}$

C T

\section{Tetrasporales}

Palmellopsidaceae Korshikov

Palmophyllum Kützing

P. crassum (Naccari) Rabenhorst

\section{Ulotrichales}

Ulotrichaceae Kützing

Ulothrix Kützing

U. flacca (Dillwyn) Thuret

\section{Phaeophilales}

Phaeophilaceae

Phaeophila Hauck

P. dendroides (P. L. Crouan et H. M. Crouan) Batters

P. tenuis (Kylin) Nielsen (38)

L $\quad \mathrm{F} \quad \mathrm{C} \quad \mathrm{T}$ 
Table I. Continued.

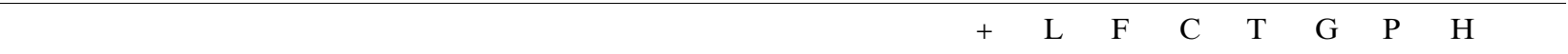

\section{Ulvales}

Gomontiaceae de Toni

Gomontia Bornet et Flahault

G. polyrhiza (Lagerheim) Bornet et Flahault

Percursariaceae Bliding

Percursaria Bory de Saint-Vincent

P. percursa (C. Agardh) Rosenvinge

C

Monostromataceae Kunieda

Blidingia Kylin

B. marginata (J. Agardh) P. Dangeard ex Bliding

B. minima (Nägeli ex Kützing) Kylin

Ulvaceae J. V. Lamouroux

Enteromorpha Link

E. clathrata (Roth) Greville

E. compressa (Linnaeus) Nees von Esenbeck

E. flexuosa (Wulfen ex Roth) J. Agardh

E. intestinalis (Linnaeus) Nees von Esenbeck

E. lingulata J. Agardh

E. linza (Linnaeus) J. Agardh

E. multiramosa Bliding

E. muscoides (Clemente) Cremades

E. prolifera (O. F. Müller) J. Agardh

E. torta (Mertens) Reinbold

Gayralia Vinogradova

G. oxysperma (Kützing) Vinogradova ex Bliding

Ulva Linnaeus

U. fasciata Delile

U. olivascens P. A. Dangeard

U. rigida C. Agardh

$U$. rotundata Bliding

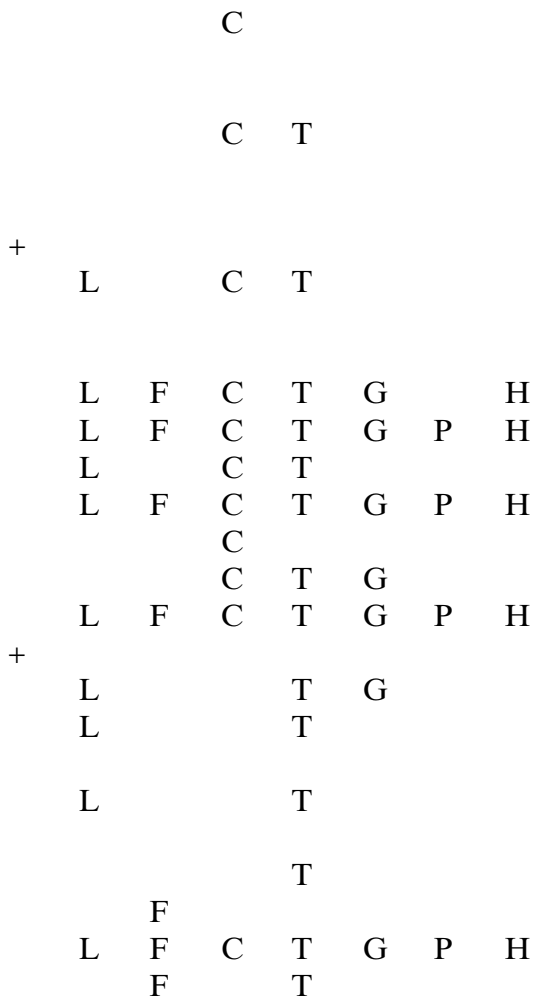

Cladophorales

Anadyomenaceae Kützing

Anadyomene J. V. Lamouroux

A. saldanhae Joly et E. C. Oliveira

A. stellata (Wulfen) C. Agardh

Microdictyon Decaisne

M. boergesenii Setchell

$M$. calodictyon (Montagne) Duchesne

M. tenuius (C. Agardh) Decaisne

Chaetosiphonaceae Blackman et Tansley

Blastophysa Reinke

B. rhizopus Reinke

Cladophoraceae Wille in Warming

Chaetomorpha Kützing

C. aerea (Dillwyn) Kützing

C. antennina (Bory de Saint-Vincent) Kützing

C. gracilis Kützing

C. ligustica (Kützing) Kützing (39)

C. linum (O. F. Müller) Kützing

C. pachynema (Montagne) Kützing

Cladophora Kützing

C. albida (Nees von Esenbeck) Kützing

C. coelothrix Kützing

C. conferta P. L. Crouan et H. M. Crouan

C. crystallina (Roth) Kützing (40)

C. cymopoliae $\mathrm{B} \varnothing$ rgesen

C. dalmatica Kützing

C. feredayi C. van den Hoek

C. flexuosa (O. F. Müller) Kützing (40)

C. hutchinsiae (Dillwyn) Kützing $\begin{array}{lllllll} & \text { F } & & \text { T } & & & \\ \text { L } & \text { F } & \text { C } & \text { T } & & \text { P } & \text { H } \\ & & & & & & \\ \text { L } & \text { F } & \text { C } & \text { T } & \text { G } & \text { P } & \text { H } \\ \text { L } & \text { F } & \text { C } & \text { T } & & & \text { H } \\ \text { L } & \text { F } & & \text { T } & & & \end{array}$

F $\quad \mathrm{T}$

$\begin{array}{lllllll}\mathrm{L} & \mathrm{F} & \mathrm{C} & \mathrm{T} & \mathrm{G} & \mathrm{P} & \mathrm{H}\end{array}$

$\mathrm{H}$

$\begin{array}{lll}\mathrm{L} & \mathrm{C} & \mathrm{T}\end{array}$

$\begin{array}{lllllll}\mathrm{L} & \mathrm{F} & \mathrm{C} & \mathrm{T} & \mathrm{G} & \mathrm{P} & \mathrm{H}\end{array}$

$\begin{array}{lllllll}\mathrm{L} & \mathrm{F} & \mathrm{C} & \mathrm{T} & \mathrm{G} & \mathrm{P} & \mathrm{H}\end{array}$

$\begin{array}{llllllll} & & \mathrm{C} & \mathrm{T} & & & \\ \mathrm{L} & \mathrm{F} & \mathrm{C} & \mathrm{T} & & \mathrm{P} & \mathrm{H}\end{array}$

L

C T

F C

L 
Table I. Continued.

\begin{tabular}{|c|c|c|c|c|c|c|c|c|}
\hline & + & $\mathrm{L}$ & $\mathrm{F}$ & $\mathrm{C}$ & $\mathrm{T}$ & G & $\mathrm{P}$ & $\mathrm{H}$ \\
\hline C. inclusa $\mathrm{B} \emptyset$ rgesen & & & & $\mathrm{C}$ & $\mathrm{T}$ & & & \\
\hline C. laetevirens (Dillwyn) Kützing & & $\mathrm{L}$ & $\mathrm{F}$ & $\mathrm{C}$ & $\mathrm{T}$ & & & $\mathrm{H}$ \\
\hline C. lehmanniana (Lindenberg) Kützing & & $\mathrm{L}$ & $\mathrm{F}$ & $\mathrm{C}$ & $\mathrm{T}$ & & & \\
\hline C. liebetruthii Grunow & & $\mathrm{L}$ & $\mathrm{F}$ & $\mathrm{C}$ & $\mathrm{T}$ & & $\mathrm{P}$ & $\mathrm{H}$ \\
\hline C. pellucida (Hudson) Kützing & & $\mathrm{L}$ & $\mathrm{F}$ & $\mathrm{C}$ & $\mathrm{T}$ & G & $\mathrm{P}$ & $\mathrm{H}$ \\
\hline C. prolifera (Roth) Kützing & & $\mathrm{L}$ & $\mathrm{F}$ & $\mathrm{C}$ & $\mathrm{T}$ & G & $\mathrm{P}$ & $\mathrm{H}$ \\
\hline C. sericea (Hudson) Kützing (40) & & & & & $\mathrm{T}$ & & $\mathrm{P}$ & \\
\hline C. vagabunda (Linnaeus) C. van den Hoek & & $\mathrm{L}$ & $\mathrm{F}$ & $\mathrm{C}$ & $\mathrm{T}$ & G & & \\
\hline \multicolumn{9}{|l|}{ Rhizoclonium Kützing } \\
\hline$R$. riparium (Roth) Kützing ex Harvey & & $\mathrm{L}$ & & $\mathrm{C}$ & $\mathrm{T}$ & & & \\
\hline R. tortuosum (Dillwyn) Kützing & & $\mathrm{L}$ & & $\mathrm{C}$ & $\mathrm{T}$ & & & \\
\hline \multicolumn{9}{|l|}{ Urospora Areschoug } \\
\hline$U$. laeta (Thuret ex Bornet) Børgesen & & & & $\mathrm{C}$ & $\mathrm{T}$ & & & \\
\hline \multicolumn{9}{|l|}{ Siphonocladaceae F. Schmitz } \\
\hline \multicolumn{9}{|l|}{ Boodlea G. Murray et de Toni } \\
\hline B. struveoides Howe & & & & & $\mathrm{T}$ & & & \\
\hline \multicolumn{9}{|l|}{ Cladophoropsis Børgesen } \\
\hline C. macromeres W. R. Taylor & & $\mathrm{L}$ & $\mathrm{F}$ & & & & & \\
\hline C. membranacea (Hofman Bang ex C. Agardh) Børgesen & & $\mathrm{L}$ & $\mathrm{F}$ & $\mathrm{C}$ & $\mathrm{T}$ & G & & $\mathrm{H}$ \\
\hline \multicolumn{9}{|l|}{ Phyllodictyon J. E. Gray } \\
\hline P. pulcherrimum J. E. Gray (41) & & $\mathrm{L}$ & $\mathrm{F}$ & $\mathrm{C}$ & $\mathrm{T}$ & & $\mathrm{P}$ & \\
\hline \multicolumn{9}{|l|}{ Siphonocladus F. Schmitz } \\
\hline S. tropicus (P. L. Crouan et H. M. Crouan) J. Agardh & & $\mathrm{L}$ & & $\mathrm{C}$ & $\mathrm{T}$ & & & \\
\hline \multicolumn{9}{|l|}{ Valoniaceae Kützing } \\
\hline \multicolumn{9}{|l|}{ Dictyosphaeria Decaisne ex Endlicher } \\
\hline D. ocellata (Howe) J. L. Olsen & & $\mathrm{L}$ & $\mathrm{F}$ & $\mathrm{C}$ & & & & \\
\hline \multicolumn{9}{|l|}{ Ernodesmis Børgesen } \\
\hline E. verticillata (Kützing) B $\varnothing$ rgesen & & $\mathrm{L}$ & & & $\mathrm{T}$ & & & $\mathrm{H}$ \\
\hline \multicolumn{9}{|l|}{ Valonia C. Agardh } \\
\hline V. aegagropila C. Agardh (42) & + & & & & & & & \\
\hline$V$. macrophysa Kützing & & $\mathrm{L}$ & $\mathrm{F}$ & $\mathrm{C}$ & $\mathrm{T}$ & & & $\mathrm{H}$ \\
\hline V. utricularis (Roth) C. Agardh & & $\mathrm{L}$ & $\mathrm{F}$ & $\mathrm{C}$ & $\mathrm{T}$ & G & $\mathrm{P}$ & $\mathrm{H}$ \\
\hline \multicolumn{9}{|l|}{ Bryopsidales } \\
\hline \multicolumn{9}{|l|}{ Bryopsidaceae Bory de Saint-Vincent } \\
\hline \multicolumn{9}{|l|}{ Bryobesia Weber-van Bosse } \\
\hline B. johannae Weber-van Bosse & & & & & & & & $\mathrm{H}$ \\
\hline Bryopsidella Feldmann & & & & & & & & \\
\hline B. neglecta (Berthold) H. Rietema & & $\mathrm{L}$ & $\mathrm{F}$ & & $\mathrm{T}$ & & $\mathrm{P}$ & $\mathrm{H}$ \\
\hline Bryopsis Laouroux & & & & & & & & \\
\hline B. corymbosa J. Agardh & & $\mathrm{L}$ & & $\mathrm{C}$ & & & $\mathrm{P}$ & \\
\hline B. cupressina J. V. Lamouroux & & $\mathrm{L}$ & $\mathrm{F}$ & & $\mathrm{T}$ & & & \\
\hline B. duplex De Notaris & & $\mathrm{L}$ & & $\mathrm{C}$ & $\mathrm{T}$ & G & & \\
\hline B. hypnoides J. V. Lamouroux & & $\mathrm{L}$ & $\mathrm{F}$ & $\mathrm{C}$ & $\mathrm{T}$ & & & \\
\hline B. plumosa (Hudson) C. Agardh & & $\mathrm{L}$ & $\mathrm{F}$ & $\mathrm{C}$ & $\mathrm{T}$ & G & & $\mathrm{H}$ \\
\hline Derbesia Solier & & & & & & & & \\
\hline D. marina (Lyngbye) Solier & & & & & $\mathrm{T}$ & & & \\
\hline D. tenuissima (Moris et De Notaris) P. L. Crouan et H. M. Crouan & & $\mathrm{L}$ & $\mathrm{F}$ & $\mathrm{C}$ & $\mathrm{T}$ & & & \\
\hline Pseudobryopsis Berthold & & & & & & & & \\
\hline P. myura (J. Agardh) Berthold (43) & & $\mathrm{L}$ & & $\mathrm{C}$ & & & & \\
\hline Caulerpaceae Kützing & & & & & & & & \\
\hline Caulerpa J. V. Lamouroux & & & & & & & & \\
\hline C. cupressoides (Vahl) C. Agardh & & $\mathrm{L}$ & $\mathrm{F}$ & & & & & \\
\hline C. mexicana Sonder ex Kützing & & $\mathrm{L}$ & $\mathrm{F}$ & $\mathrm{C}$ & $\mathrm{T}$ & & & $\mathrm{H}$ \\
\hline C. prolifera (Forsskål) J. V. Lamouroux & & $\mathrm{L}$ & $\mathrm{F}$ & $\mathrm{C}$ & $\mathrm{T}$ & G & $\mathrm{P}$ & \\
\hline C. racemosa (Forsskål) J. Agardh (44) & & $\mathrm{L}$ & $\mathrm{F}$ & $\mathrm{C}$ & $\mathrm{T}$ & G & $\mathrm{P}$ & $\mathrm{H}$ \\
\hline C. sertularioides (S. G. Gmelin) Howe & & & & & $\mathrm{T}$ & & & \\
\hline C. webbiana Montagne & & $\mathrm{L}$ & $\mathrm{F}$ & $\mathrm{C}$ & $\mathrm{T}$ & $\mathrm{G}$ & $\mathrm{P}$ & $\mathrm{H}$ \\
\hline Codiaceae Kützing & & & & & & & & \\
\hline Codium Stackhouse & & & & & & & & \\
\hline C. adhaerens C. Agardh & & $\mathrm{L}$ & $\mathrm{F}$ & $\mathrm{C}$ & $\mathrm{T}$ & G & $\mathrm{P}$ & $\mathrm{H}$ \\
\hline C. bursa (Linnaeus) C. Agardh & & $\mathrm{L}$ & $\mathrm{F}$ & $\mathrm{C}$ & & & & \\
\hline
\end{tabular}


Table I. Continued.

\begin{tabular}{|c|c|c|c|c|c|c|c|c|}
\hline & + & $\mathrm{L}$ & $\mathrm{F}$ & $\mathrm{C}$ & $\mathrm{T}$ & $\mathrm{G}$ & $\mathrm{P}$ & $\mathrm{H}$ \\
\hline C. carolinianum Searles & & $\mathrm{L}$ & $\mathrm{F}$ & & & & & \\
\hline C. decorticatum (Woodward) Howe & & $\mathrm{L}$ & $\mathrm{F}$ & $\mathrm{C}$ & $\mathrm{T}$ & $\mathrm{G}$ & & $\mathrm{H}$ \\
\hline C. effusum (Rafinesque) Delle Chiaje & & $\mathrm{L}$ & $\mathrm{F}$ & & $\mathrm{T}$ & & $\mathrm{P}$ & $\mathrm{H}$ \\
\hline C. elisabethiae O. C. Schmidt (45) & & $\mathrm{L}$ & & & & & & \\
\hline C. fragile (Suringar) Hariot (46) & & $\mathrm{L}$ & $\mathrm{F}$ & & & & & \\
\hline C. guineënse P. Silva ex Lawson et John & & & & & $\mathrm{T}$ & & & \\
\hline C. intertextum Collins et Hervey & & $\mathrm{L}$ & $\mathrm{F}$ & $\mathrm{C}$ & $\mathrm{T}$ & $\mathrm{G}$ & $\mathrm{P}$ & $\mathrm{H}$ \\
\hline C. repens P. L. Crouan et H. M. Crouan ex Vickers & & $\mathrm{L}$ & & $\mathrm{C}$ & & & & \\
\hline C. taylorii $\mathrm{P}$. Silva & & $\mathrm{L}$ & $\mathrm{F}$ & $\mathrm{C}$ & $\mathrm{T}$ & $\mathrm{G}$ & $\mathrm{P}$ & $\mathrm{H}$ \\
\hline C. tomentosum Stackhouse (47) & & & & & $\mathrm{T}$ & & & \\
\hline C. vermilara (Olivi) Delle Chiaje & & $\mathrm{L}$ & $\mathrm{F}$ & & $\mathrm{T}$ & & & \\
\hline \multicolumn{9}{|l|}{ Halimedaceae Link } \\
\hline \multicolumn{9}{|l|}{ Halimeda J. V. Lamouroux } \\
\hline H. discoidea Decaisne & & $\mathrm{L}$ & $\mathrm{F}$ & $\mathrm{C}$ & & & & \\
\hline H. tuna (J. Ellis et Solander) J. V. Lamouroux & & $\mathrm{L}$ & $\mathrm{F}$ & $\mathrm{C}$ & & & & \\
\hline \multicolumn{9}{|l|}{ Ostreobiaceae } \\
\hline \multicolumn{9}{|l|}{ Ostreobium Bornet et Flahault } \\
\hline O. quekettii Bornet et Flahault & & $\mathrm{L}$ & & $\mathrm{C}$ & & & & \\
\hline \multicolumn{9}{|l|}{ Udoteaceae J. Agardh } \\
\hline \multicolumn{9}{|l|}{ Avrainvillea Decaisne } \\
\hline A. canariensis A. Gepp et E. S. Gepp & & & & $\mathrm{C}$ & $\mathrm{T}$ & & & \\
\hline \multicolumn{9}{|l|}{ Flabellia Reichenbach } \\
\hline F. petiolata (Turra) Nizamuddin & & $\mathrm{L}$ & $\mathrm{F}$ & & & & & \\
\hline \multicolumn{9}{|l|}{ Pseudochlorodesmis Børgesen } \\
\hline P. furcellata (Zanardini) Børgesen & & $\mathrm{L}$ & $\mathrm{F}$ & $\mathrm{C}$ & $\mathrm{T}$ & $\mathrm{G}$ & $\mathrm{P}$ & $\mathrm{H}$ \\
\hline \multicolumn{9}{|l|}{ Dasycladales } \\
\hline \multicolumn{9}{|l|}{ Dasycladaceae Kützing } \\
\hline \multicolumn{9}{|l|}{ Batophora J. Agardh } \\
\hline B. occidentalis (Harvey) S. Berger et Kaever ex M. J. Wynne (48) & & & & & $\mathrm{T}$ & & & \\
\hline \multicolumn{9}{|l|}{ Cymopolia J. V. Lamouroux } \\
\hline C. barbata (Linnaeus) J. V. Lamouroux & & $\mathrm{L}$ & $\mathrm{F}$ & $\mathrm{C}$ & $\mathrm{T}$ & & & \\
\hline \multicolumn{9}{|l|}{ Dasycladus C. Agardh } \\
\hline D. vermicularis (Scopoli) Krasser & & $\mathrm{L}$ & $\mathrm{F}$ & $\mathrm{C}$ & $\mathrm{T}$ & G & $\mathrm{P}$ & $\mathrm{H}$ \\
\hline \multicolumn{9}{|l|}{ Polyphysaceae Kützing } \\
\hline \multicolumn{9}{|l|}{ Acetabularia J. V. Lamouroux } \\
\hline A. acetabulum (Linnaeus) P. Silva & & $\mathrm{L}$ & & $\mathrm{C}$ & & & & \\
\hline A. calyculus J. V. Lamouroux & & $\mathrm{L}$ & & & $\mathrm{T}$ & & & \\
\hline A. parvula Solms-Laubach & & & $\mathrm{F}$ & $\mathrm{C}$ & $\mathrm{T}$ & & $\mathrm{P}$ & \\
\hline A. polyphysoides P. L. Crouan et H. M. Crouan & & $\mathrm{L}$ & $\mathrm{F}$ & $\mathrm{C}$ & $\mathrm{T}$ & G & $\mathrm{P}$ & $\mathrm{H}$ \\
\hline \multicolumn{9}{|l|}{ MAGNOLIOPHYTA } \\
\hline \multicolumn{9}{|l|}{ LILIOPSIDA } \\
\hline \multicolumn{9}{|l|}{ Alismatales } \\
\hline Hydrocharitaceae A. L. de Jussieu & & & & & & & & \\
\hline Halophila Du Petit-Thouars & & & & & & & & \\
\hline H. decipiens Ostenfeld & & & & $\mathrm{C}$ & $\mathrm{T}$ & & $\mathrm{P}$ & \\
\hline Zosterales & & & & & & & & \\
\hline Cymodoceaceae N. Taylor & & & & & & & & \\
\hline Cymodocea K. D. König & & & & & & & & \\
\hline C. nodosa (Ucria) Ascherson & & $\mathrm{L}$ & $\mathrm{F}$ & $\mathrm{C}$ & $\mathrm{T}$ & $\mathrm{G}$ & $\mathrm{P}$ & $\mathrm{H}$ \\
\hline Zosteraceae Dumortier & & & & & & & & \\
\hline Zostera Linnaeus & & & & & & & & \\
\hline Z. noltii Horneman (49) & & $\mathrm{L}$ & & & & & & \\
\hline
\end{tabular}

\section{MYCOPHYTA}

ASCOMYCOTA

Dothideales

Arthopyreniaceae W. Watson

Arthopyrenia A. Massalongo

A. halodytes (Nylander) Arnold 
Table I. Continued.

+ L F C T G P H

Leptosphaeriaceae M. E. Barr

Leptosphaeria Cesati et De Notaris

L. oraemaris Linder

Mycosphaerellaceae Lindau

Stigmidium Trevisan

S. balani (Winter) Bauch

Pleosporaceae Nitschke

Kirschteiniothelia D. Hawksworth

K. maritima (Linder) D. Hawksworth

Halosphaeriales

Halosphaeriaceae E. Müller et von Arx ex Kohlmeyer

Arenariomyces Höhnk ex E. B. G. Jones

A. trifurcatus Höhnk ex E. B. G. Jones, R. G. Johnson et T. S. Moss

Ceriosporopsis Linder

C. halima Linder

$\mathrm{T}$

Chadefaudia Feldmann-Mazoyer

C. corallinarum (P. L. Crouan et H. M. Crouan) E. Müller et von Arx

Corollospora Werdermann

C. maritima Werdermann

Haligena Kohlmeyer

H. elaterophora Kohlmeyer

Halosphaeria Linder

$H$. appendiculata Linder

H. quadriremis (Höhnk) Kohlmeyer

Lulworthia G. K. Sutherland

L. kniepii Kohlmeyer

Nautosphaeria E. G. B. Jones

N. cristaminuta E. G. B. Jones

L $\quad \mathrm{F} \quad \mathrm{T}$

$\mathrm{H}$

\section{Lecanorales}

Dactylosporaceae Hafellner et Bellemère

Dactylospora Körber

D. canariensis Kohlmeyer et Volkmann-Kohlmeyer

\section{Lichinales}

Lichinaceae Nylander

Lichina C. Agardh

L. confinis (O. F. Müller) C. Agardh

L $\quad$ F

$\mathrm{T}$

\section{Sordariales}

Lasiosphaeriaceae Nannfeldt

Chaetosphaeria R. L. Tulasne et C. Tulasne

C. chaetosa Kohlmeyer

\section{Ascomycota incertida sedis}

Thalassoascus Ollivier

T. cystoseirae (Ollivier) Kohlmeyer $\quad$ T

T. tregoubovii Ollivier $\quad \mathrm{T}$

Torpedospora Meyers

T. radiata Meyers

\section{Fungi imperfecti}

Cirrenalia Meyers et R. T. Moore

C. macrocephala (Kohlmeyer) Meyers et R. T. Moore $\quad$ T

Zalerion R. T. Moore et Meyers

Z. maritimum (Linder) Anastasiou

Z. varium Anastasiou 


\section{Notes}

(1) The systematic position of these taxa is mainly in accordance with the recent publications of Komárek and Anagnostidis (1995, 1999).

(2) Gil-Rodríguez and Afonso-Carrillo (1980a: 23) reported this species as Anacystis dimidiata (Kützing) Drouet et Daily, including the earlier record of Frémy (1936:11-12). Later González-Ruíz et al. (1995: 15) reported it for Fuerteventura Island and Afonso-Carrillo and Sansón (1999: 27) included this species in their recent key.

(3) Aphanocapsa orae (Kosinskaja) Komárek et Anagnostidis is a superfluous name for A. littoralis Hansgirg. The later is the correct name under Aphanocapsa. Thus, A. littoralis (Hansgirg) Komárek et Anagnostidis based on Polycystis littoralis Hansgirg is a later homonym of $A$. littoralis Hansgirg and has to be renamed.

(4) Aphanocapsa marina (Hansgirg) P. Silva (see in P. Silva et al. 1996) cannot be considered the correct name. It ought to be $A$. marina Hansgirg in Foslie. The alternative combination Microcystis marina (Hansgirg) P. Silva is a later homonym of Microcystis marina (Hansgirg) Kosinskaja.

(5) This species was reported by Reyes et al. (1993: 55, figs 18-20) growing on rocks and epiphytic on other algal species, such as Fucus spiralis.

(6) These two Scinaia species, S. australis and S. caribaea, were reported for the first time from the area by Reyes et al. (1993: 55-57, figs 21-22) mainly from subtidal habitats.

(7) This species is considered conspecific with L. fragilis as reported by John et al. (1994: 58), following the criteria of Abbott (1990a: 312, fig. 3). Nevertheless, further studies are needed to support the view of Silva et al. (1996:127-128) who considered that both entities are not conspecific.

(8) This species was described by Grunow as Gelidium cartilagineum var. canariensis from material collected at Puerto Orotava (Tenerife) and Gran Canaria by Liebetruth (see Piccone 1884: p. 56). Later, Seoane-Camba (1979) renamed it as $G$. canariensis (Grunow in Piccone) Seoane-Camba, and Prud'homme van Reine et al. (1994) accepted that proposal. Nevertheless, in accordance with article 33.3 of the ICBN (Greuter et al. 2000) this combination was not correctly done and therefore we validated this new name by a full and direct reference to its author and place of valid publication: Gelidium canariense (Grunow in Piccone) Seoane-Camba ex Haroun, Gil-Rodríguez, Díaz de Castro et Prud'homme van Reine comb. nov. Basionym: Gelidium cartilagineum var. canariensis Grunow in Piccone (1884: 56) collected at Orotava, Gran Canaria. G. canariensis: SeoaneCamba, Acta Bot. Malacit., 5, 1979: p. 106, nom. illeg.

(9) According to Price et al. (1988) often cited for the
Canary Islands. Nevertheless, no voucher specimen could be found until now. Recently, this species was collected on a field trip to the northern coast of Gran Canaria Island by J. Rico and $\mathrm{R}$. Haroun. Material has been deposited in BCM (No. 3609). Details of this new record will be published elsewhere.

(10) Includes records as G. spinulosum (C. Agardh) J. Agardh.

(11) The first record of this species in the Canary Islands was as G. ferox J.Agardh. See González Henríquez (1991) and Oliveira et al. (1983).

(12) Gracilaria gracilis (Stackhouse) Steentoft, L. M. Irvine et Farnham is an illegitimate name for $G$. confervoides (L.) Greville. It also falls within the original description of $G$. verrucosa (Hudson) Papenfuss, of which another part belongs to Gracilariopsis longissima (S. G. Gmelin) Steentoft, L. M. Irvine et Farnham. This is, however, also a illegitimate name and deserves a new name.

(13) Includes the records of Gracilaria rubra (C. Agardh) J. Agardh by Gil-Rodríguez and Afonso-Carrillo (1980a), which is probably a misspelling of G. dura (C. Agardh) J. Agardh.

(14) This species was quoted by Tabares et al. (1997) and by Afonso-Carrillo and Sansón (1999: 199) without providing any additional information of its distributional pattern.

(15) The sporophytic phase: Falkenbergia rufolanosa (Harvey) Schmitz, is also reported. The same applies to the sporophytic phase: F. hillebrandii (Bornet) Falkenberg, which is also found in the Canary Islands. It is not possible to separate the species based only on the morphology of the Falkenbergia stages.

(16) Only the sporophytic phase: Trailliella intricata Batters has been reported in the islands. See Prud'homme van Reine (1998).

(17) The sporophytic phase of this species, Rhododiscus pulcherrimus P. L. Crouan et H. M. Crouan, has been collected growing on shells of Patella sp. See Gil-Rodríguez et al. (1985).

(18) Cited in the Canaries as S. ornata Schousboe ex Kützing and recently collected in the islets located north of Lanzarote Island. Details of these records will be published elsewhere.

(19) The record of H. squamatum by Price et al. (1992: 125) needs confirmation.

(20) The only record of this adelphoparasite is in Haroun and Prud'homme van Reine (1993), with three voucher specimens deposited at $\mathrm{L}$ with the numbers 1307 and 1315 on Hypnea spinella (C. Agardh) Kützing, and 6146 on Hypnea valentiae (Turner) Montagne, from the CANCAP Expeditions.

(21) This family was erected to accomodate multiaxial genera formerly placed in Nemastomataceae, 
such as Schizymenia and Platoma. See Masuda and Guiry (1995).

(22) The genus Sebdenia is being revised in the Macaronesian region; Soler-Onís et al. (1996) and published elsewhere.

(23) This species was recently reported by AfonsoCarrillo and Sansón (1999: 50, 203, fig. 15) for the Canary Islands, without specifying any particular location or island.

(24) This species was also reported by Afonso-Carrillo and Sansón (1999: 50, 203, fig. 15) questioning the presence of $A$. elegans (Berthold) J. H. Price et D. M. John in the Canary Islands. Nevertheless, recent collections do not support their view.

(25) The Canarian material is probably all C. poiteaui (J. V. Lamouroux) K. W. Nam. Both species are retained here until further data are collected to support it.

(26) This northern species was quoted by AfonsoCarrillo and Sansón (1999) and Rojas-Gónzalez and Afonso-Carrillo (2000b: 122) as occurring in the Canary Islands, including the older records of $P$. myriococca Montagne and P. nutans Montagne for Gran Canaria Island. The correct synonym might well be $P$. fucoides (Hudson) Greville $[=P$. violacea (Roth) Sprengel].

(27) Rojas-Gónzalez and Afonso-Carrillo (2000a) have recently found this interesting rhodomelacean species in subtidal habitats with a certain degree of siltation.

(28) The systematic position of this supposed adelphoparasite is still unclear; see Rojas-González and Afonso-Carrillo (2000a).

(29) Ectocarpus repens Reinke does not have a clear taxonomic position. According to some authors it is Chilonema reptans (P. L. Crouan et H. M. Crouan) Sauvageau, or it is considered to be a microstage of Asperococcus fistulosus (Hudson) Hooker, a species occasionally recorded for the Canary Islands (amongst others by Fletcher, 1987: 298).

(30) The combination Hapalospongidion macrocarpum for Mesospora macrocarpa (Feldmann) Hartog was made by León-Álvarez and González-González (1993: 474) in a frequently overlooked Mexican publication, following the proposal of Womersley (1987: 74-75) to merge the genera Basispora, Hapalospongidium and $\mathrm{Me}$ sospora under the earliest name, Hapalospongidium.

(31) This species was recorded by Vickers (1905: 58) from Gran Canaria. After that time, only Afonso-Carrillo and Sansón (1999: 114, 210, fig. 7a) and Sansón et al. (2002) confirmed its presence also in Tenerife Island.

(32) These three Dictyota species are only quoted by Afonso-Carrillo and Sansón (1999: 111-112, 211, figs 5a, 5i and 5l), but without specification of any particular island. Besides, Hörnig et al. (1992b) considered them not to be present in the Eastern Atlantic Ocean.

(33) The real number of species belonging to this genus in the Canary Islands remains to be determined (as well as in the other Macaronesian Archipelagos).

(34) The first record of C. peregrina (Sauvageau) Hamel is credited to Johnston (1969) from Lanzarote Island. Later, Audiffred (1984: 166, figs 1-2) and Reyes and Sansón (1990: 77, fig. 1) reported it from El Hierro Island. However, the presence of this cold-water species needs to be confirmed.

(35) The record of this species is credited to Fletcher (1987: 298). See remarks under (28).

(36) This species was recently described from limited Canarian material by Nizamuddin (1995), but the taxonomic relationship of this new entity with other Cystoseira species present in nearby areas remains to be determined.

(37) This species was reported by Afonso-Carrillo and Sansón (1999) for the Canary Islands, without specifying any particular island.

(38) Phaeophila tenuis was recently reported for the Canary Islands, as Entocladia tenuis (Kylin) Nielsen, by Afonso-Carrillo and Sansón (1999: pp. 122-123, 213, figs 5, 13a) without specifying any particular island.

(39) Includes $C$. capillaris (Kützing) Børgesen and $C$. mediterranea (Kützing) Kützing recently reported by Afonso-Carrillo and Sansón (1999: 125, 214).

(40) Cladophora crystallina (Roth) Kützing and $C$. flexuosa (O. F. Müller) Kützing are probably synonyms of $C$. sericea (Hudson) Kützing. However, according to Hoek (1982) the latter species does not occur south of mainland Spain. New data are needed to discern the taxonomic status of the Canarian specimens.

(41) According to Børgesen (1925: 72), the Canarian specimens [Struvea anastomosans (Harvey) Piccone et Grunow var. canariensis Piccone] are conspecific with Struvea ramosa Sonder, which it is now quoted as Phyllodictyon pulcherrimum based on Kraft and Wynne (1996: 139). Nevertheless, a critical review of these two genera along their pantropical distribution range is needed.

(42) Valonia aegagropila C. Agardh was reported by Afonso-Carrillo and Sansón (1999) for the Canary Islands without specifying any particular island. The morphological plasticity of Valonia species, such as the widespread $V$. utricularis (Roth) C. Agardh, may hamper an accurate identification. Thus, this species is retained here until a detailed study of this genus is made on the Eastern Atlantic coasts.

(43) Henne and Schnetter (1999) recognized Pseudobryopsis and Trichosolen as separate genera, based on considerable differences in chloroplast features and gametangial behaviour. 
(44) Caulerpa racemosa includes different varieties, such as var. laetevirens, var. occidentalis, var. lamourouxii and var. peltata (sometimes reported also as $C$. peltata J. V. Lamouroux). The experimental evidence obtained by Ohba and Enomoto (1987) strongly supports the merge of these varieties as morphological extremes in response to a gradient in light intensity.

(45) This species collected near Montaña Clara (Lanzarote) by Prud'homme van Reine during a CANCAP Expedition, 31-III-83 (L, 8143), was reported by Audiffred and Prud'homme van Reine (1985: 28).

(46) Chacana (1990) identified as C. fragile ssp. tomentosoides (van Goor) P. Silva some specimens attached to ropes inside two small fishing/recreational harbours.

(47) Several records from other Canarian Islands are currently considered as misidentification of similar species, such as C. decorticatum (Woodward) Howe and $C$. taylorii P. Silva (Chacana, pers. comm.).

(48) Sterile plants were collected in a tidepool of El Medano (Tenerife Island) by Reyes et al. (1993: 50-51, fig. 1) as B. oerstedii J. Agardh var. occidentalis (Harvey) M. Howe.

(49) At present, endangered populations of this species are only found in very restricted coastal areas of Lanzarote Island. See Guadalupe-González et al. (1995) and Pavón-Salas et al. (2000).

\section{List of Taxa Inquirenda}

Several doubtful records of macroalgae from the Canary Islands are included here in alphabetic order as there is no recent information to support their presence. Some other records such as Ahnfeltia plicata (Hudson) Fries, Antithamnion pteroton Schousboe ex Bornet, Callophyllis fastigiata (J. Agardh) J. Agardh, Chrondrus crispus Stackhouse, Phymatolithon bornetii (Foslie) Foslie, Porphyra atropurpurea (Olivi) De Toni, Ascophyllum nodosum (Linnaeus) Le Joly, Ecklonia muratii Feldmann, Cystoseira nodicaulis (Whitering) Roberts, Rhipilia tenaculosa A. Gepp et E. Gepp, Ulva uvoides Bory de Saint-Vincent have been rejected or considered as taxa inquirenda by GilRodríguez and Afonso-Carrillo (1980) and AfonsoCarrillo and Sansón (1999).

\section{Cyanophycota}

Aphanocapsa orae (Kosinskaja) Komárek et Anagnostidis. This species was incidentally reported for the Canary Islands by Komárek and Anagnostidis (1999: 160) without mentioning any collecting station. Its presence needs to be confirmed.

Bachytrichia maculans Gomont. Lawson and Norton (1971) reported this species from the northern coast of Tenerife Island. From the information pro- vided in their publication, it seems that it may correspond to Calothrix crustacea Schousboe et Thuret ex Bornet et Flahault. Thus, this record needs to be verified.

Blennothrix cantharidosma (Montagne ex Gomont) Anagnostidis et Komárek. Since the first collection by Despréaux (and reported by Montagne as Lyngbya cantharidosma in Webb et Berthelot 1840: p. 188), this species has not been found in the area.

Chamaecalyx leibleiniae (Reinsch) Komárek et Anagnostidis. Frémy (1936: 16) reported this species [as Dermocarpa leibleiniae (Reinsch) Bornet et Thuret] from a single sample in Gran Canaria Island. No other recent record is available.

Chamaecalyx swirenkoi (Sirsov) Komarek et Anagnostidis. This species was reported by Kristiansen et al. (1993: 94) in culture growing on Anotrichum sp. from Lanzarote Island. Nevertheless, according to Komarek and Anagnostidis (1999: 401) this is a freshwater species. This record, however, might be listed under the name Ch. clavatus (Setchell et Gardner) Komarek et Anagnostidis.

Gomphosphaeria aponina Kützing. First reported by Frémy (1936: 10-11) from Bahía del Confital, Gran Canaria. Later, it was reported again from the same locality by González (1976: 62). This species was listed by Gil-Rodríguez and Afonso-Carrillo (1980a: 23) and also by Afonso-Carrillo and Sansón (1999: 27, 193). Nevertheless, this name applies to a freshwater species.

Hormothamnion enteromorphoides (Grunow) ex Bornet et Flahault. Frémy (1936: 40-41) did not study this species and only reported it as observed by Vickers between November and February as a rare epiphytic species on Spyridia at Bahía del Confital (Gran Canaria).

Leptolyngbya fragilis (Meneghini ex Gomont) Anagnostidis et Komárek. This species was cited with doubt by Frémy (1936: 22-23) from an isolated sample (No. 3985) collected in Gran Canaria:...'Christoballo, ? Phormidium fragile Gomont, avec les espèces mentionnées à propos de Aphanocapsa marina Hangsg'. Further samples are needed to confirm its presence.

Lyngbya polychroa (Meneghini) Rabenhorst. There is only one incidental record of this species, as Lyngbya sordida (Zanardini) ex Gomont, by Ballesteros (1992: fig. 1, tab. 2) from a deep water area off Lanzarote.

Lyngbya semiplena (C. Agardh) ex Gomont. The only record of this species is in Johnston (1969) from coastal areas of Lanzarote Island. Nevertheless, it has not been possible to confirm this record as there is no voucher specimen available.

Merismopedia convoluta De Brébisson was reported only by Frémy (1936: 8) from a coastal area in Gran Canaria Island. This species is considered as freshwater species and thus, its status in the Canarian coasts needs to be reviewed.

Merismopedia thermalis Kützing. Although this species occurs in thermal water only, several records 
of this species [as Agmenellum thermale (Kütz.) Drouet et Daily] are reported for the Canary Islands, such as Pinedo et al. (1991: 31) and Afonso-Carrillo and Sansón (1999: 27).

Oscillatoria angina (Børgesen) Gomont and O. limosa (Dillwyn) C. Agardh ex Gomont were also reported by Johnston (1969) from coastal areas in Lanzarote Island. Nevertheless, it has not been possible to confirm these records as there are no voucher specimens available.

Rivularia monticulosa Montagne ex Bornet et Flahault. The only record of this species is by Sauvageau (1912), who mentioned this species incidentally in his study of Cystoseira species from the Canarian coast. No other records exist.

\section{Rhodophycota}

Acrochaetium codii (P. L. Crouan et H. M. Crouan) G. Hamel. This species was first reported for the Canary Islands by Feldmann in 1946; other authors such as Ardré (1970: 10) and Levring (1974: 52) cited it as Rhodothamniella codii, and Price et al. (1986: 20) as Audouinella codii (P. L. et H. M. Crouan) Garbary, without adding any new records. Its presence in the Canary Islands requires confirmation.

Cordylecladia erecta (Greville) J. Agardh. The only voucher specimen assigned to this species is deposited in L (CANCAP II Expedition, 15-IX-1977, No. 682), from the intertidal area of Pto. de Arinaga (Gran Canaria). Besides, Ballesteros (1992: fig. 1, tab. I) mentioned this species from a maërl bottom area in Fuerteventura. According to Brodie and Guiry (1988), this species seems to be confined to the north eastern Atlantic Ocean. A review of the Canarian samples is needed to verify if these are conspecific with the Mediterranean C. guiryi Gargiulo, Furnari et Cormaci (Gargiulo et al. 1990).

Dumontia canariensis Montagne. In the description of this species, Montagne (1840: 165) did not include any illustration, neither did Børgesen (1929) who was able to study the holotype and two small fragments from the original collection of Despréaux, but did not arrive at any conclusion regarding its identity. The taxonomic status of this entity still remains uncertain.

Halurus flosculosus (J. Ellis) Maggs et Hommersand was reported for the islands as Griffithsia setacea C. Agardh sensu Montagne in Webb et Berthelot (1840: 175). According to Børgesen (1930: 30-31), the material is not present in Herb. Montagne in the Muséum National d'Histoire Naturelle in Paris.

Heterosiphonia pellucida was only reported by Johnston (1969), and subsequently cited by other authors without any new data. Its presence in the islands needs confirmation.

Jania micrarthrodia J. V. Lamouroux was cited by Price et al. (1992: 136). It has not been possible to locate authentical material for this record.

Kallymenia perforata J. Agardh was cited by Price $e t$ al. (1992) based on a pers. comm. by Prud'homme van Reine on 10 April 1987 to J. H. Price from a dredge sample south of Lanzarote (CANCAP IV Expedition, station 4.072, 45-60 m, 20-5-1980), but no further information has been published yet. The correct species might well be $K$. westii Ganesan, a species from the western Atlantic coasts.

Lithophyllum crouanii Foslie was reported for the Canaries only by John et al. (1994: 61) and needs confirmation.

Lithophyllum daedaleum Foslie et Howe was cited for the Canaries by John et al. (1994: 61). Although the holotype has been recently studied in detail in a modern context (Woelkerling and Lamy 1998: 308-309, figs 169-170), the identification of the material from the Canary Islands is still uncertain.

Lithophyllum decussatum (Ellis et Solander) Philippi f. planiscula Foslie was reported by John et al. (1994: 61). No such specimen is filed in the Foslie herbarium under Lithophyllum decussatum (Adey and Lebednik 1967: 44), and until the relevant specimen is located and examined in a modern context, this record should be regarded as questionable.

Lithophyllum papillosum (Zanardini) Price, John et Lawson reported by Woelkerling et al. (1998: 128) based on the records of Lithophyllum (Dermatolithon) papillosum (Zanardini) Foslie by Lemoine (1929: 46-47) for Tenerife and Gran Canaria is considered as a doubtful species requiring confirmation by Afonso-Carrillo et al. (1983: 46).

The report of Mesophyllum brachycladum (Foslie) W. H. Adey by Prud'homme van Reine [in John et al. (1994: 73)] requires confirmation. It might be Lithophyllum racemus (Lamarck) Foslie.

Mesophyllum erubescens (Foslie) Lemoine. This species was reported incidentally for the Canary Islands by Lemoine (1964) in her study of the coralline algae from the Cape Verde Archipelago. It needs confirmation.

Polysiphonia erythraea (Schousboe) J. Agardh, was reported by Afonso-Carrillo and Sansón (1999) without specification of any particular island; its presence needs confirmation.

Ptilota gunneri P. Silva, Maggs et L. M. Irvine ex Maggs et Hommersand [ $=P$. plumosa (Hudson) C. Agardh]. This species was reported as Fucus plumosus Linnaeus by Bory de Saint-Vicent (1803: 304) for the Canary Islands. According to Børgesen (1930) and Gil-Rodríguez and Afonso-Carrillo (1980a: 12, 33) this was a misidentification of Spyridia hypnoides (Bory de Saint-Vicent) Papenfuss.

\section{Chromophycota}

Two Asperococcus species reported from Gran Canaria Island are considered here as dubious records: $A$. bullosus J. V. Lamouroux (by Børgesen 1926: 68-69) and $A$. compressus Griffiths ex Hooker (only cited by Afonso-Carrillo and Sansón 1999: 119, 209, fig. 11). 
Further data are needed to clarify their taxonomic value.

Myriotrichia canariensis Kützing was reported for the islands without any indication of the locality by Kützing (1856) and De Toni (1895). Only small fragments of the original material remain in $\mathrm{L}$, which seems very similar to $M$. adriatica Hauck. The taxonomic value of this entity remains to be determined.

Sphacelaria racemosa Greville, a boreal species, does most probably not occur in the Canary Islands. It was only recorded by González (1976: 64) from Playa de las Canteras, Gran Canaria.

\section{Chlorophycota}

Caulerpa taxifolia (Vahl) C. Agardh was reported by Lawson and Price (1969: 291-292) and subsequently other authors mentioned it for different islands. Afonso-Carrillo et al. (1983: 42), after reviewing the TFC herbarium, considered that all specimens were C. mexicana Sonder ex Kützing.

Cladophoropsis monodensis (Kützing) Reichembach. This species was cited only by Meñez and Mathieson (1981: 28) in their study of the Tunisian algae. New data are needed to confirm its presence in the Canary Islands.

Spongomorpha aeruginosa (Linnaeus) van den Hoek, was reported only by Bory de Saint-Vincent (1803: 306), growing on rocks on Santa Cruz Bay

\section{References}

Abbott, I. A. 1990a. A taxonomic assessment of the species of Liagora (Nemaliales, Rhodophyta) recognized by J. Agardh, based upon studies of type specimens. Cryptogamic Bot. 1: 308-322.

Abbott, I. A. 1990b. A taxonomic and nomenclatural assessment of the species of Liagora (Rhodophyta, Nemaliales) in the herbarium J. V. Lamouroux. Cryptogamie, Algol. 11: $111-136$

Adey, W. H. and P. A. Lebednik. 1967. Catalog of the Foslie Herbarium. Trondheim, Norway. Det Kongelige Norske Videnskabers Selskab Museet. pp. 92.

Afonso-Carrillo, J. 1980. Nota sobre algunas Corallinaceae (Rhodophyta) nuevas para la flora ficológica de las islas Canarias. Vieraea 10: 53-58.

Afonso-Carrillo, J. 1983. Estudios en las algas Corallinaceae (Rhodophyta) de las Islas Canarias. II. Notas taxonómicas. Vieraea 13: 127-144.

Afonso-Carrillo, J. 1988. Structure and reproduction of Spongites wildpretii sp. nov. (Corallinaceae, Rhodophyta) from the Canary Islands, with observations and comments on Spongites absimile comb. nov. Br. phycol. J. 23: 89-102.

Afonso-Carrillo, J. and M. C. Gil-Rodríguez. 1982. Sobre la presencia de un fondo de "maerl" en las islas Canarias. Collectanea Botánica 13: 703-708.

Afonso-Carrillo, J. and M. Sansón. 1999. Algas, hongos y fanerógamas marinas de las Islas Canarias. Clave analítica. Servicio de Publicaciones Universidad de La Laguna, La Laguna. pp. 256.

Afonso-Carrillo, J., M. C. Gil-Rodríguez and W. Wildpret de
(Tenerife Island). Since that time no other collections have been added. Gil-Rodríguez and Afonso-Carrillo (1980a: 2, 27) considered it as a doubtful record, but Afonso-Carrillo and Sansón (1999: 130, 213) still retained this species without providing any evidence of its presence.

Ulva lactuca Linnaeus. Several old records of this species are found in the literature (Bory de SaintVincent 1803: 306, Montagne 1840: 181, Vickers 1896: 298, Frémy 1936: 27) and, subsequently, later authors include the Canaries in its distribution range (Feldmann 1946: 403, Lawson and Price 1969: 334335). Gil-Rodríguez and Afonso-Carrillo (1980a: 14) considered these records as misidentifications of $U$. rigida $\mathrm{C}$. Agardh, although it is present in the northern Madeira Archipelago (Neto et al. (2001: 393).

\section{Acknowledgements}

The final layout of this paper has been enriched by inputs and suggestions of many colleagues. We feel much indebted to Professors I. A. Abbott, M. D. Guiry, P.C. Silva and W. J. Woelkerling for their accurate comments on the taxonomic status and recent nomenclatural changes of several taxa. Also, the technical help of Mr M. Garrido (ULPGC) during typing is much appreciated.

Accepted 28 November 2001.

la Torre. 1978. Estudio de la vegetación algal de la costa del futuro polígono industrial de Granadilla (Tenerife). Vieraea 8: 201-242.

Afonso-Carrillo, J., M. C. Gil-Rodríguez, R. Haroun Tabraue, M. Villena Balsa and W. Wildpret de la Torre. 1983. Adiciones y correcciones al catálogo de algas marinas bentónicas para el Archipiélago Canario. Vieraea 13: 27-49.

Afonso-Carrillo, J., S. Pinedo and Y. Elejabeitia. 1992. Notes on the benthic marine algae of the Canary Islands. Cryptogamie, Algol. 13: 281-290.

Afonso-Carrillo, J., M. Sansón and J. Reyes. 1998. Vegetative and reproductive morphology of Ganonema lubrica sp. nov. (Liagoraceae, Rhodophyta) from the Canary Islands. Phycologia 37: 319-329.

Anagnostidis, K. and J. Komárek. 1988. Modern approach to the classification system of cyanophytes 3 - Oscillatoriales. Arch. Hydrobiol. Suppl. Band 80: 327-472, figs 1-35, tabs $1-13$.

Anagnostidis. K. and J. Komárek. 1990. Modern approach to the classification system of cyanophytes 5 - Stigonematales. Arch. Hydrobiol. Suppl. Band 85: [1]-73, figs 1-14, tabs $1-4$.

Ardré, F. 1970. Contribution a l'etude des algues marines du Portugal I. La Flore. Port. Acta Biol. B 10: 137-155.

Audiffred, P. A. J. 1984. Marine algae of El Hierro (Canary Islands). Vieraea 14: 157-183.

Audiffred, P. A. J. and W. F. Prud'homme van Reine. 1985. Marine algae of Ilha do Porto Santo and Deserta Grande 
(Madeira Archipelago). Bol. Mus. Munic. Funchal 37: 2051.

Ballesteros, E. 1992. Algunas observaciones sobre las comunidades de algas profundas en Lanzarote y Fuerteventura (Islas Canarias). Vieraea 22: 17-27.

Ballesteros, E., M. Sansón, J. Reyes, J. Afonso-Carrillo and M. C. Gil-Rodríguez. 1992. New records of benthic marine algae from the Canary Islands. Bot. Mar. 35: 513-522.

Betancor Villalba, M. ${ }^{a}$ J., M. N. González Henríquez, R. Haroun Tabraue, R. Herrera Pérez, E. Soler Onís and M. A. Viera Rodríguez. 1995. Adiciones corológicas a la flora marina de Canarias. Bot. Macaronésica 22: 75-89.

Børgesen, F. 1925-30. Marine algae from the Canary Islands especially from Teneriffe and Gran Canaria. I. Chlorophyceae. II. Phaeophyceae. III. Rhodophyceae, part I: Bangiales and Nemalionales. III. Rhodophyceae, part II: Cryptonemiales, Gigartinales and Rhodymeniales. III. Rhodophyceae, part III: Ceramiales. Biologiske Meddelelser. Kgl. Danske Videnskabernes Selskab. - Bind 5, 3: 1-123. Bind 6, 2:1-112. Bind 6, 6:1 -97. Bind 8, 1:1-97. Bind 9, 1:1-159.

Bory de Saint-Vincent, J. B. G. M. 1803. Essais sur les Isles Fortunées et l'antique Atlantide, ou Précis de l'histoire générale de l'Archipel des Canaries. Paris: Baudouin, ..., Paris. [i-viii], [1]-522, [2 p. err.] pp., pls. 1-7.

Brodie, J. and M. D. Guiry. 1988. Life history and reproduction of Cordylecladia erecta (Rhodymeniales, Rhodophyta). Br. phycol. J. 23: 347-364.

Brummitt, R. K. and C. E. Powell. 1992. Authors of Plant Names: a List of Scientific Names of Plants, with Recommended Standard Forms of their Names, Including Abbreviations. Royal Bot. Garden, Kew. London. pp. 732.

Chacana, M. 1990. Ocurrencia de Codium fragile ssp.tomentosoides en Canarias. Abstract First Congress on Oceanography and Marine Resources in the Central-East Atlantic, Cabildo de Gran Canaria, Taliarte (Gran Canaria): p. 109.

De Toni, G.-B. 1895. Sylloge Algarum Omnium Hucusque Cognitarum. Vol 3. Sylloge Fucoidearum. Padova, xvi + pp. 638.

Feldmann, J. 1946. La flore marine des îles Atlantides. Mémoires de la Société de Biogéographie 8: 395-435.

Fletcher, R. L. 1987. Seaweeds of the British Isles... Volume 3. Fucophyceae (Phaeophyceae). Part 1. British Museum (Natural History), London. $\mathrm{x}+359$ pp., 90 figs, 15 pls, 3 tabs.

Frémy, P. 1936. Marine algae from the Canary Islands: especially from Teneriffe and Gran Canaria IV. Cyanophyceae collected by F. Børgesen, worked out by P. Frémy. Biologiske Meddelelser. Kgl. Danske Videnskabernes Selskab, Bind 12, no. 5: 1-43.

Gallardo, T., A. Gómez-Garreta, M. A. Ribera, M. Cormaci, G. Furnari, G. Giaccone and Ch. F. Boudouresque. 1993. Check-list of Mediterranean seaweeds II. Chlorophyceae Wille s. l. Bot. Mar. 36: 399-421.

Gargiulo, G. M., G. Furnari and M. Cormaci. 1990. Structure and reproduction of Cordylecladia guiryi sp. nov. (Rhodophyta, Rhodymeniales) from the Mediterranean Sea. Giorn. Bot. Ital. 124: 631-640.

Gil-Rodríguez, M. C. and J. Afonso-Carrillo. 1980a. Catálogo de las Algas Marinas Bentónicas (Cyanophyta, Chlorophyta, Phaeophyta y Rhodophyta) para el Archipiélago Canario. Aula de Cultura de Tenerife. Santa Cruz de Tenerife, pp. 47.

Gil-Rodríguez, M. C. and J. Afonso-Carrillo. 1980b. Adiciones a la flora marina y catálogo ficológico para la isla de Lanzarote. Vieraea 10: 59-70.
Gil-Rodríguez, M. C. and R. Haroun. 1992. Laurencia viridis sp. nov. (Ceramiales, Rhodophyta) from the Macaronesian Archipelagos. Bot. Mar. 35: 227-237.

Gil-Rodríguez, M. C. and R. Haroun. 1993. The genus Laurencia (Rhodomelaceae, Rhodophyta) in the Canary Islands. Courier Forschungsinstitut Senckenberg. 159: 113117.

Gil-Rodríguez, M. C., R. Haroun Tabraue, J. Afonso-Carrillo and W. Wildpret de la Torre. 1985. Adiciones al catálogo de las algas marinas bentónicas para el Archipiélago Canario. II. Vieraea 15: 101-112.

González, N. 1976. Contribución al estudio del epifitismo en Zostera marina L. (Zosteraceae) en la Playa de Las Canteras (Gran Canaria). Bot. Macaronésica 2: 59-67.

González Henríquez, M. N. 1991. Gracilaria ferox J. Agardh, nuevo taxon para la isla de Gran Canaria. Acta Bot. Malacitana 16: 59-62.

González-Ruíz, S., M. Sansón and J. Reyes. 1995. New records of sublittoral algae from the Canary Islands. Cryptogamie, Algol. 16: 21-31.

Greuter, W., J. McNeill, F. R. Barrie, H. M. Burdet, V. Demoulin, T.S. Filgueiras, D. H. Nicolson, P.C. Silva, J. E. Skog, P. Trethane, N. J. Turland and D. L. Hawksorth. 2000. International Code of Botanical Nomenclature (Saint Louis Code). Koeltz Scientific Books, Königstein. 474 pp.

Guadalupe, M. E., M. C. Gil-Rodríguez and M. C. Hernández-González. 1995. Fitobentos de Arrecife de Lanzarote, reserva de la biosfera (Islas Canarias). Cryptogamie, Algol. 16: 33-46.

Guiry, M. D. 1997. Benthic red, brown and green algae. In: (C. M. Howson and B. E. Picton, eds): The species directory of the marine fauna and flora of the British Isles and surrounding seas. Ulster Museum Publication 276:341-367.

Haroun, R. J. and W. F. Prud'homme van Reine. 1993. A biogeographical study of Laurencia and Hypnea species of the Macaronesian Region. Courier Forschungsinstitut Senckenberg. 159: 119-125.

Haroun, R. J., W. F. Prud'homme van Reine, D. G. Müller, E. Serrao and R. Herrera. 1993. Deep-water macroalgae from the Canary Islands: new records and biogeographical relationships. Helgoländer Meeresunters. 47: 125-143.

Hawksworth, D. L., P. M. Kirk, B. C. Sutton and D. N. Pegler. 1995. Ainsworth \& Bisby's Dictionary of the Fungi. 8th edition. International Mycological Institute, Wallingford, U. K., CAB International. xii, pp. 616.

Henne, K.-D. and R. Schnetter, 1999. Revision of the Pseudobryopsis/Trichosolen complex (Bryopsidales, Chlorophyta) based on features of gametangial behaviour and chloroplasts. Phycologia 38: 114-127.

Hoek, C. van den. 1982. A Taxonomic Revision of the American Species of Cladophora (Chlorophyceae) in the North Atlantic Ocean and Their Geographic Distribution. NorthHolland Publ. Co., Amsterdam. pp. 236.

Holmgren, P. K., N. H. Holmgren and L. C. Barnett. 1990. Index Herbariorum. Part I. The herbaria of the world. 8th edition. Regnum Vegetabile 120: 1-693.

Hörnig, I., R. Schnetter and W.F. Prud'homme van Reine. 1992a. The genus Dictyota (Phaeophyceae) in the North Atlantic. I. A new generic concept and new species. Nova Hedwigia 54: 45-62.

Hörnig, I., R. Schnetter and W. F. Prud'homme van Reine. 1992b. The genus Dictyota (Phaeophyceae) in the North Atlantic. II. Key to the species. Nova Hedwigia 54: 397402 . 
Hörnig, I., R. Schnetter and W. F. Prud'homme van Reine. 1993. Additional notes to "The genus Dictyota (Phaeophyceae) in the North Atlantic. I. A new generic concept and new species." Correction and validation of new combinations in the genus Dictyota. Nova Hedwigia 56: 169-171.

John, D. M., G. W. Lawson, J. H. Price, W. F. Prud'homme van Reine and W. J. Woelkerling. 1994. Seaweeds of the western coast of tropical Africa and adjacent islands: a critical assessment. IV. Rhodophyta (Florideae) 4. Genera L-O. Bull. Nat. Hist. Mus. London (Bot. Ser.) 24: 49-90.

Johnston, C. S. 1969. The ecological distribution and primary productivity of macrophytic marine algae in the Eastern Canaries. Internat. Rev. Ges. Hydrobiol. 54: 473-490.

Jong, Y. S. D. M. de and W. F. Prud'homme van Reine. 1997. A review of the genus Nemacystus (Sporochnaceae, Chordariales, Phaeophyceae), including phylogenetic and biogeographical hypotheses. Nova Hedwigia 64: 1-40.

Jong, Y. S. D. M. de, W. F. Prud'homme van Reine and G. M. Lokhorst. 1997. Studies on Dasyaceae. 2. A revision of the genera Eupogodon and Dipterocladia gen. nov. (Ceramiales, Rhodophyta). Bot. Mar. 40: 421-450.

Kim, M. S. and I. K. Lee. 1999. Neosiphonia flavimarina gen. et $\mathrm{sp}$. nov. with a taxonomic reassessment of the genus Polysiphonia (Rhodomelaceae, Rhodophyta). Phycol. Res. 47: 271-281.

Komárek, J. and K. Anagnostidis. 1995. Nomenclatural novelties in chroococcalean cyanoprokaryotes. Preslia 67: $15-23$.

Komárek, J. and K. Anagnostidis. 1999. Cyanoprokaryota 1. Teil: Chroococcales. In: Süßwasserflora von Mitteleuropa, Band 19/1. Gustav Fischer, Jena. pp. 1-548, figs 1-643.

Kraft, G. and M. J. Wynne. 1996. Delineation of the genera Struvea Sonder and Phyllodictyon J. E. Gray (Cladophorales, Chlorophyta). Phycol. Res. 44: 129-142.

Kristiansen, A., R. Nielsen, and P. M. Pedersen. 1993. An annotated list of marine algae collected on Lanzarote, Canary Islands, January 1986. Courier Forschungsinstitut Senckenberg. 159: 93-102.

Kützing, F. T. 1856. Tabulae Phycologicae Vol. 6. Nordhausen. ii +35 pp., 100 pls.

Kvaternik, D. and J. Afonso-Carrillo. 1995. The red algal genus Liagora (Liagoraceae, Rhodophyta) from the Canary Islands. Phycologia 34: 449-471.

Lawson, G. W. and D. M. John. 1987. The marine algae and coastal environment of tropical west Africa. 2nd edition. Beih. Nova Hedwigia 93: 1-415.

Lawson, G. W. and T. Norton. 1971. Some observations on littoral and sublittoral zonation at Teneriffe (Canary Islands). Bot. Mar. 14: 116-120.

Lawson, G. W. and J. H. Price. 1969. Seaweeds of the western coast of tropical Africa and adjacent islands: a critical assessment. I. Chlorophyta and Xanthophyta. Bot. J. Linn. Soc. 62: 279-346.

Lemoine, P. 1929. Subfamilia 1. Melobesieae. In: (F. Børgesen, ed.) Marine algae from the Canary Islands: especially from Teneriffe and Gran Canaria. III. Rhodophyceae, part II: Cryptonemiales, Gigartinales and Rhodymeniales. Biol. Meddelelser. Kgl. Danske Videnskabernes Selskab 8: 19-68.

Lemoine, P. 1964. Contribution a l'étude des Mélobésiées de'l Archipel du Cap-Vert. Proc. Int. Seaweed Symposium 4: 234-239.

León-Álvarez, D. and J. González-González. 1993. Algas costrosas del Pacífico tropical. 456-474. In: (S. I. Salazar
Vallejo and N. E. González, eds) Biodiversidad Marina y Costera de México. Chetumal, Mexico. 865 pp.

Levring, T. 1974. The marine algae of the archipelago of Madeira. Bolm. Mus. munic. Funchal 28: 5-111.

Martín, M. J., M. Sansón and J. Reyes. 1996. Morphology and anatomy of Papenfussiella kuromo (Chordariaceae, Phaeophyta) from the Canary Islands. Cryptogamie, Algol. 17: 165-173.

Masuda, M. and M. D. Guiry. 1995. Reproductive morphology of Itonoa marginifera (J. Agardh) gen. et comb. nov. (Nemastomataceae, Rhodophyta). Eur. J. Phycol. 30: 5767.

Meñez, E. G. and A. C. Mathieson. 1981. The marine algae of Tunisia. Smith. Contrib. Mar. Sc. 10: 1-59.

Montagne, J. P. F. C. 1840. Plantae cellulares. In: (P. Webb and S. Berthelot, eds) Histoire naturelle des îles Canaries, Tome troisième. Deuxième partie: Phytographia canariensis. Sectio ultima. Béthune, París. [4] + xv + [1] + 208 pp., Láms 1-9.

Morales-Ayala, S. and M. A. Viera-Rodríguez. 1988. Adiciones al catálogo de las algas marinas bentónicas para el archipiélago canario. Vieraea 18: 189-192.

Nam, K. W. 1999. Morphology of Chondrophycus undulata and $C$. parvipapillata and its implications for the taxonomy of the Laurencia (Ceramiales, Rhodophyta) complex. Eur. J. Phycol. 34: 455-468.

Nam, K. W., C. A. Maggs and D. J. Garbary. 1994. Resurrection of the genus Osmundea with an emendation of the generic delimitation of Laurencia (Ceramiales, Rhodophyta). Phycologia 33: 384-395.

Nam, K. W., C. A. Maggs, L. McIvor and M. J. Stanhope. 2000. Taxonomy and phylogeny of Osmundea (Rhodomelaceae, Rhodophyta) in Atlantic Europe.J. Phycology, 36: 759772.

Neto, A. I., D. C. Cravo and R. J. Haroun. 2001. Check-list of the benthic marine plants of the Madeira Archipelago. Bot. Mar. 44: 391-414.

Nielsen, R., A. Kristiansen, L. Mathiesen and H. Mathiesen. 1995. Distributional index of the benthic macroalgae of the Baltic Sea area. Acta Bot. Fennica 155: 1-51.

Nizamuddin, M. 1995. Cystoseira wildpretii Nizamuddin sp. nov. from the Canary Islands. Pakistan J. Bot. 27: 263-266.

Ohba, H. and S. Enomoto. 1987. Culture studies on Caulerpa (Caulerpales, Chlorophyceae) II. Morphological variation of $C$. racemosa var. laetevirens under various culture conditions. Jap. J. Phycol. 35: 178-188.

Oliveira, F. E. C. de, C. J. Bird and J. McLachlan. 1983. The genus Gracilaria Greville (Rhodophyta, Gigartinales): The western Atlantic G. domingensis Sonder ex Kütz., pro syn., G. cervicornis (Turner) J. Ag. and G. ferox J. Ag. Can J. Bot. 61: 2999-3008.

Pavón-Salas, N., R. Herrera, A. Hernández-Guerra and R. Haroun. 2000. Distribution pattern of seagrasses in the Canary Islands (Central-East Atlantic Ocean). J. Coastal Res. 16: 329-335.

Pedersen, P. M. and A. A. Kristiansen. 1995. Temperature gradient experiments on Giraudia sphacelarioides (Algae, Fucophyceae) from Lanzarote, Canary Islands. Bolm. Mus. munic. Funchal Supl. 4B: 589-597.

Piccone, A. 1884. Crociera del Corsaro alle Isole Madera e Canarie del capitano Enrico d'Albertis. Alghe. Tipografia del R. Istituto Sordo-Muti, Genova. pp. 60, 1 lám.

Price, J. H., D. M. John and G. W. Lawson. 1986. Seaweeds of the western coast of tropical Africa and adjacent islands: a critical assessment. IV. Rhodophyta (Florideae) 1. Genera 
A-F. Bull. Brit. Mus. (Nat. Hist.), Bot. Ser. 15: 1-122.

Price, J. H., D. M. John and G. W. Lawson. 1988. Seaweeds of the western coast of tropical Africa and adjacent islands: a critical assessment. IV. Rhodophyta (Florideae) 2. Genera G. Bull.Brit. Mus. (Nat. Hist.), Bot. Ser. 18: 195-273.

Price, J. H., D. M. John and G. W. Lawson. 1992. Seaweeds of the western coast of tropical Africa and adjacent islands: a critical assessment. IV. Rhodophyta (Florideae) 3. Genera H-K. Bull. Brit. Mus. (Nat. Hist.), Bot. Ser. 22: 123-146.

Prud'homme van Reine, W. F. 1998. Seaweeds and biogeography in the Macaronesian Region. Bolm. Mus. munic. Funchal Supl. 5B: 307-331.

Prud'homme van Reine, W. F. and C. van den Hoek. 1990. Biogeography of Macaronesian seaweeds. Courier Forschungsinstitut Senckenberg 129: 55-73.

Prud'homme van Reine, W. F., R. J. Haroun and P. A. J. Audiffred. 1994. A reinvestigation of Macaronesian seaweeds as studied by A. Piccone with remarks on those studied by A. Grunow. Nova Hedwigia 58: 67-121.

Reyes, J. and M. Sansón. 1990. Adiciones a la flora marina de la isla de El Hierro (Islas Canarias). Vieraea 20: 71-81.

Reyes, J., M. Sansón and J. Afonso-Carrillo. 1993. Notes on some interesting marine algae new from the Canary Islands. Cryptogamic Bot. 4: 50-59.

Ribera, M. A., A. Gomez-Garreta, T. Gallardo, M. Cormaci, G. Furnari and G. Giaccone. 1992. Check-list of Mediterranean Seaweeds I. Fucophyceae (Warming, 1884). Bot. Mar. 35: 109-130.

Rojas-González, B. and J. Afonso-Carrillo. 2000a. Notes on Rhodomelaceae (Rhodophyta) from the Canary Islands: Observations on reproductive morphology and new records. Bot. Mar. 43: 147-155.

Rojas-González, B. and J. Afonso-Carrillo. 2000b. Notas corológicas sobre algas rojas Rhodomelaceae de las islas Canarias. Vieraea 28: 119-125.

Rojas-González, B. and J. Afonso-Carrillo. 2000c. Morfología y distribución de Boergeseniella fruticulosa en las islas Canarias (Rhodophyta, Rhodomelaceae). Vieraea 28: 127-136.

Rojas-González, B., J. Afonso-Carrillo and C. Ibeas. 1994. New records of Rhodomelaceae (Rhodophyta) from the Canary Islands. Bot. Mar. 37: 133-138.

Sansón, M. and J. Reyes. 1994. Notes on Ceramiaceae (Rhodophyta) from the Canary Islands: new records and observations on morphology and geographical distribution. Bot. Mar. 37: 347-356.

Sansón, M. and J. Reyes. 1995. Morphological and geographical observations on four species of Ceramiaceae (Rhodophyta) new to the Canary Islands. Bot. Mar. 38: 89-95.

Sansón, M., J. Reyes and J. Afonso-Carrillo. 1991. Contribution to the seaweed flora of the Canary Islands: new records of Florideophyceae. Bot. Mar. 34: 527-536.

Sansón, M., J. Reyes, J. Afonso-Carrillo and E. Muñoz. 2002.
Sublittoral and deep-water red and brown algae new from the Canary Islands. Bot. Mar. 45: 35-49.

Sauvageau, C. 1912. A propos des Cystoseira de Banyuls et Guéthary. Bull. Stn. Biol. Arcachon 14: 133-556.

Seoane-Camba, J. 1979. Sobre algunas Gelidiaceas nuevas o poco conocidas de las costas españolas. Acta Bot. Malacitana 5: 99-112.

Silva, P. C. 1992. Geographic patterns of diversity in benthic marine algae. Pacific Science 46: 429-437.

Silva, P. C., P. W. Basson and R. L. Moe. 1996. Catalogue of the benthic marine algae of the Indian Ocean. Univ. California Publ. Bot. 79: xiv + 1-1281.

Soler-Onís, E., R. J. Haroun, M. A. Viera-Rodríguez, W.F. Prud'homme van Reine and Y.S. D. M. de Jong. 1996. Systematics and phylogenetics of the genus Sebdenia Berthold in the Atlantic Ocean. In: (R. Haroun and M. A. Viera-Rodríguez, eds) II Symposium Fauna and Flora Atlantic Islands. Univ. Las Palmas de Gran Canaria. 137 pp.

South, G. R. and I. Tittley. 1986. A Check-list and Distributional Index of the Benthic Marine Algae of the North Atlantic Ocean. Huntsman Marine Laboratory and British Museum (Natural History), St. Andrews and London. 76 pp.

Tabares, N., J. Afonso-Carrillo, M. Sansón and J. Reyes. 1997. Vegetative and reproductive morphology of Dudresnaya canariensis sp. nov. (Dumontiaceae, Rhodophyta). Phycologia 36: 267-273.

Thorne, R. F. 1992. Classification and geography of the flowering plants. The Botanical Review 58: 225-348.

Vickers, A. 1896. Contribution a la flora algologique des Canaries. Annls. Sc. Nat. (Bot.), ser. 8, 4: 293-306.

Vickers, A. 1905. Liste de algues marines de la Barbade. Annls. Sc. Nat. (Bot.), ser. 9, 1: 45-66.

Viera-Rodríguez, M. A., P. A. J. Audiffred, M. C. Gil-Rodríguez, W. F. Prud'homme van Reine and J. Afonso-Carrillo. 1987. Adiciones al catálogo de algas marinas bentónicas para el Archipiélago Canario. III. Vieraea 17: 227-235.

Woelkerling, W. J. and D. Lamy. 1998. Non geniculate Coralline Red Algae and the Paris Muséum: Systematics and Scientific History. Publ. Scientifiques du Muséum/ A. D. A. C., Paris. viii +767 pp., 369 pls.

Woelkerling, W. J., G. W. Lawson, J. H. Price, D. M. John and W. F. Prud'homme van Reine. 1998. Seaweeds of the western coast of tropical Africa and adjacent islands: a critical assessment. IV. Rhodophyta (Florideae) 5. Genera [Q] RZ. Bull. Nat. Hist. Mus. London (Bot. Ser.) 28: 115-150.

Womersley, H. B. S. 1987. The Marine Benthic Flora of Southern Australia. Part II. South Australian Government Printing Division, Adelaide. 484 pp., 169 figs, 8 pls, 1 tab.

Wynne, M. J. 1998. A Check-list of Benthic Marine Algae of the Tropical and Subtropical Western Atlantic: First Revision. Beih. Nova Hedwigia 116: iii + pp. 155. 